Richter, C. (Ed.)

Proceedings of the Ocean Drilling Program, Scientific Results Volume 181

\title{
4. Data Report: Utilizing Color Reflectance Analysis as a Carbonate Concentration Proxy 1
}

\author{
Lynn D. Millwood, ${ }^{2}$ D.L. Hawkins, Jr., ${ }^{3}$ and Shana M. Wells ${ }^{2}$
}

\section{INTRODUCTION}

Color reflectance data $(400-700 \mathrm{~nm})$ are routinely collected aboard the JOIDES Resolution and have been used to estimate the carbonate concentration in sediments (Balsam et al., 1997; Mix et al., 1995). A good summary of the recent usage of color reflectance data to estimate fractions of various minerals is published in Balsam et al. (1999).

The purpose of this paper is to present models estimating the carbonate fraction for each site cored during Leg 181. These models ("Appendix A," p. 6) were constructed using data collected aboard ship during Leg 181 (hereafter referred to as "shipboard data") and supplementary data collected at the Ocean Drilling Program Gulf Coast Repository (ODP GCR) after the cruise (hereafter referred to as "laboratory data"). Each model is contained in a separate table and includes predicted carbonate fractions with corresponding depth values in meters composite depth (mcd) (Carter, McCave, Richter, Carter, et al., 1999). Graphic depictions for each model are included in "Appendix B," p. 7.

\section{METHODS AND MATERIALS}

Leg 181 was the maiden voyage for the automated system now used to collect color reflectance data. At the first site of Leg 181, Site 1119, the Minolta spectrophotometer did not consistently make good contact with core material, which resulted in poor data quality data for Site 1119 and left the shipboard color reflectance data unusable. Adjustments were made in the settings of the spectrophotometer before data

${ }^{1}$ Millwood, L.D., Hawkins, D.L., Jr., Wells, S.M., 2002. Data report: Utilizing color reflectance analysis as a carbonate concentration proxy. In Richter, C. (Ed.), Proc. ODP, Sci. Results, 181, 1-50 [Online]. Available from World Wide Web: <http://www-odp. tamu.edu/publications/181_SR/ VOLUME/CHAPTERS/208.PDF $>$. [Cited YYYY-MM-DD]

${ }^{2}$ Department of Geology, University of Texas at Arlington, PO Box 19049, Arlington TX 76019, USA.

Correspondence author:

Idm8510@omega.uta.edu

${ }^{3}$ Department of Mathematics, University of Texas at Arlington, PO Box 19408, Arlington TX 76019, USA.

Initial receipt: 5 December 2001

Acceptance: 5 March 2002

Web publication: 3 December 2002

Ms 181SR-208 
were collected at subsequent sites, and therefore data quality for the remaining cores is better (Carter, McCave, Richter, Carter, et al., 1999). Color reflectance data (laboratory data) were collected on cores from Hole 1119C at the ODP GCR with a Minolta CM-2002 spectrophotometer. This is the same model instrument used aboard ship. The software package used in the data collection process was provided by the manufacturer. A barium sulfate standard was used. Cores were covered with Glad plastic wrap before taking measurements to protect the optical components of the instrument from moisture and scratches and to avoid sample contamination. A glass slide was used to scrape mold growth from core surfaces. Measurements were taken on cores by careful visual inspection to ensure good contact with the core material and avoid confounders such as cracks, large shell fragments, pebbles, and pyrite oxidation halos.

Figure $\mathbf{F} 1$ is a comparative display of the ranges of color reflectance data (all wavelengths combined) for each hole. The maximum, $75^{\text {th }}$ quartile, median, $25^{\text {th }}$ quartile, and minimum values are displayed in a box and whisker format. There are obvious problems with the data. Cores from Site 1120 show out-of-range values for Holes 1120B and $1120 \mathrm{C}$ (reflectance $>100 \%$ ). A comparison with the measured carbonate summary statistics (Table T1) shows that these values are significantly higher at Site 1120 than at the other sites. These problematic data were not discarded because no other color reflectance data from this site are available. Because the measured carbonate values for Site 1120 are higher than those from other sites and because the range of color reflectance values is similar to data from other cores, we utilized these data to construct the model for Site 1120. Interquartile range values for color reflectance data from Site 1120 are similar to those of other cores recovered during this leg, which suggests the possibility that they preserve some of the variance in the data set, even though absolute values are not reliable.

Models to estimate carbonate concentrations were generated using the statistics package produced by the SAS Institute, Inc., and are regression models. Models for Sites 1124 and 1125, and Holes 1119C and $1123 \mathrm{~A}$ used the following model form:

$$
Y=\beta_{0}+\beta_{1} \mathrm{FD}_{1}+\beta_{2} \mathrm{FD}_{2}+\ldots .+\beta_{30} \mathrm{FD}_{30} .
$$

The model for Site 1121 has the form

$$
Y=\beta_{0}+\beta_{1} R_{1}+\beta_{2} \mathrm{FD}_{4}+\beta_{3} \mathrm{FD}_{7}+\beta_{4} \mathrm{FD}_{21}+\beta_{5} \mathrm{FD}_{30}
$$

The model for Site 1120 has the form

$$
Y=\beta_{0}+\beta_{1} R_{1}+\beta_{2} \mathrm{FD}_{2}+\beta_{3} \mathrm{FD}_{7}+\beta_{4} \mathrm{FD}_{21}+\beta_{5} \mathrm{FD}_{27}
$$

Models for Sites 1122 and 1123 (all shipboard data) are of the form

$$
Y=\beta_{0}+\beta_{1} R_{1}+\beta_{2} \mathrm{FD}_{1}+\beta_{3} \mathrm{FD}_{2}+\ldots .+\beta_{30} \mathrm{FD}_{30} .
$$

For all these models,

$Y=$ the dependent variable, predicted carbonate fraction.

$\beta_{\mathrm{i}}=$ the coefficient (model parameter) estimated in the modeling process.
F1. Ranges of color reflectance data, p. 10.

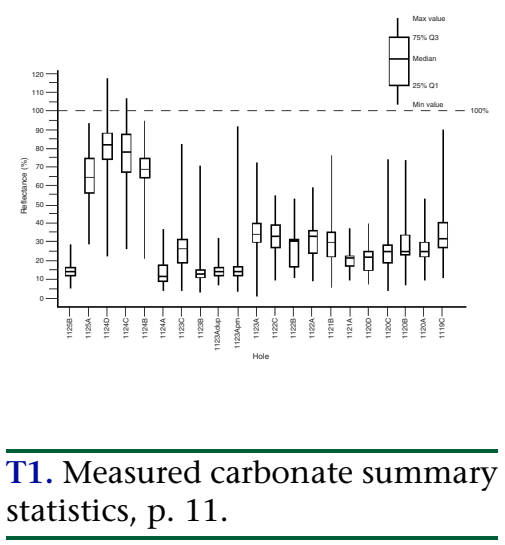


L.D. MILLWOOD ET AL.

$R_{1}=$ the raw reflectance value at $400 \mathrm{~nm}$ wavelength.

$\mathrm{FD}_{\mathrm{i}}=$ the first derivative value calculated using two successive reflectance values (such as $\mathrm{FD}_{1}$ calculated from $R_{1}[400 \mathrm{~nm}]$ and $R_{2}$ [410 nm]).

Development of the models proceeded empirically. The choice of first derivative transformation of the reflectance data was suggested by the work of Deaton and Balsam (1991) and the collective experience of the authors. The data were analyzed to minimize the covariance problem that is inherent in reflectance data. Variables were selected based on this study and various combinations of the least covariant factors were tried in order to choose empirically the most effective regression model. Adding the first untransformed reflectance variable $\left(R_{1}=400 \mathrm{~nm}\right)$ helped increase the $R^{2}$ value for some of the models. An indication of how well each model describes the data can be ascertained by inspecting Table T2. Models that have low enough $R^{2}$ values $(<50 \%)$ to be of questionable value as prediction tools are the models for Sites 1122 and 1123. Several of the models account well for most of the variation in the data-namely, the models for Sites 1121 and 1125 and for Hole 1119C. Plots of measured carbonate vs. predicted carbonate fraction can be examined for every model in "Appendix C," p. 8. Model parameter estimates for every model are also included in "Appendix D," p. 9.

In an effort to get an indication of the relative data quality between shipboard data and laboratory data, some cores from Hole 1123A were also measured in the laboratory after the cruise. Figure F1 shows that the data range for laboratory data is not significantly different from that of the shipboard data. However, the $R^{2}$ values for the models are significantly different. The model generated using the laboratory data is significantly better than that using the shipboard data. Both models use the same variables, the same model structure, and the same depth range over the cores. This suggests that there may be a problem with the shipboard data in this interval, making it more noisy, masking the true variance of the data, and hindering the ability of the model to reflect the true variability of the data.

\section{CONCLUSIONS}

Shipboard color reflectance data contain some unresolved problems; however, most data can be used with caution. Shipboard color reflectance data from Site 1119 are not usable. The carbonate prediction model fit is good at Sites 1120, 1121, 1124, and 1125 (shipboard data) and Holes 1119C and 1123A (laboratory data). Models for Sites 1122 and 1123 (excluding the model from laboratory data) are of questionable usefulness but are included for comparison and completeness.

\section{ACKNOWLEDGMENTS}

This research used samples and/or data provided by Ocean Drilling Program (ODP). ODP is sponsored by the U.S. National Science Foundation (NSF) and participating countries under management of Joint Oceanic Institutions (JOI), Inc. Funding for this research was provided by JOI/USSP grant No. 26-3900-66. Other shipboard participants generously shared carbonate data, which they collected during postcruise research. L. Joseph contributed carbonate data for Sites 1122 and 1124. J.
T2. Carbonate prediction model statistics, p. 12. 


\section{L.D. MILLWOOD ET AL.}

Rinna was the first to provide carbonate data analyzed from Site 1122. I. Hall allowed the use of carbonate data collected from Site 1123. K. Grant supplied data collected from Site 1125. The authors are grateful for their invaluable assistance, without which these models would only be sparsely anchored to the truth. Other persons who made significant contributions to this endeavor include Karen Graber, Phil Rumford, and the ODP GCR laboratory staff. 


\section{L.D. MILLWOOD ET AL.}

\section{REFERENCES}

Balsam, W.L., Damuth, J.E., and Schneider, R.R., 1997. Comparison of shipboard vs. shore-based spectral data from Amazon-Fan cores: implications for interpreting sediment composition. In Flood, R.D., Piper, D.J.W., Klaus, A., and Peterson, L.C. (Eds.), Proc. ODP, Sci. Results, 155: College Station, TX (Ocean Drilling Program), 193-215.

Balsam, W.L., Deaton, B.C., and Damuth, J.E., 1999. Evaluating optical lightness as a proxy for carbonate content in marine sediment cores. Mar. Geol., 161:141-153.

Carter, R.M., McCave, I.N., Richter, C., Carter, L., et al., 1999. Proc. ODP, Init. Repts., 181 [CD-ROM]. Available from: Ocean Drilling Program, Texas A\&M University, College Station, TX 77845-9547, U.S.A.

Deaton, B.C., and Balsam, W.L., 1991. Visible spectroscopy: a rapid method for determining hematite and goethite concentrations in geological materials. J. Sediment. Petrol., 61:628-632.

Mix, A.C., Harris, S.E., and Janecek, T.R., 1995. Estimating lithology from nonintrusive reflectance spectra: Leg 138. In Pisias, N.G., Mayer, L.A., Janecek, T.R., PalmerJulson, A., and van Andel, T.H. (Eds.), Proc. ODP, Sci. Results, 138: College Station, TX (Ocean Drilling Program), 413-427. 


\section{APPENDIX A}

\section{Models for Leg 181 Data: Carbonate Fraction Predictions}

Models were developed to estimate the carbonate fraction for each site of Leg 181. Each of the following ASCII tables contains predicted carbonate fractions with the corresponding depth values in meters composite depth (mcd): Table ATA1 contains data for Hole 1119C; Table ATA2 contains data for Site 1120; Table ATA3 contains data for Site 1121; Table ATA4 contains data for Site 1122; Table ATA5 contains data for Site 1123; Table ATA6 contains laboratory data for Hole 1123A; Table ATA7 contains shipboard data for Hole 1123A; Table ATA8 contains data for Site 1124; and Table ATA9 contains data for Site 1125.
ATA1. Carbonate fraction prediction model, Hole 1119 C, p. 33.

ATA2. Carbonate fraction prediction model, Site 1120, p. 34.

ATA3. Carbonate fraction prediction model, Site 1121, p. 35 .

ATA4. Carbonate fraction prediction model, Site 1122, p. 36.

ATA5. Carbonate fraction prediction model, Site 1123, p. 37.

ATA6. Carbonate fraction prediction model, Hole 1123A (laboratory data), p. 38.

ATA7. Carbonate fraction prediction model, Hole 1123A (shipboard data), p. 39 .

ATA8. Carbonate fraction prediction model, Site 1124, p. 40.

ATA9. Carbonate fraction prediction model, Site 1125, p. 41. 


\section{APPENDIX B}

\section{Plots of Model Predictions: Carbonate Fraction}

The Leg 181 models for carbonate fractions were plotted. For results of the plots, see Figures AFB1, AFB2, AFB3, AFB4, AFB5, AFB6, and AFB7.

AFB1. Model-predicted carbonate fraction, Hole 1119C, p. 13.

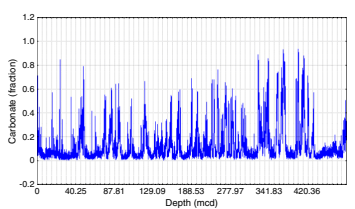

AFB2. Model-predicted carbonate fraction, Site 1120, p. 14.

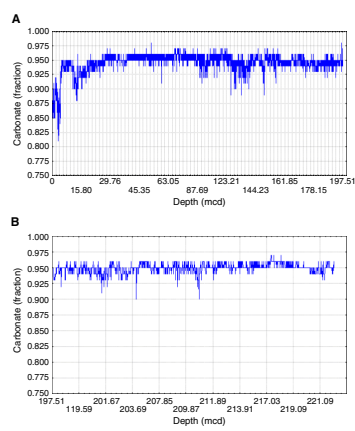

AFB3. Model-predicted carbonate fraction, Site 1121, p. 15.

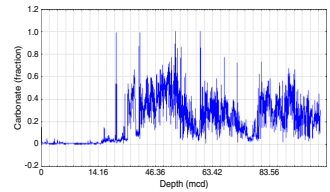

AFB4. Model-predicted carbonate fraction, Site 1122, p. 16.
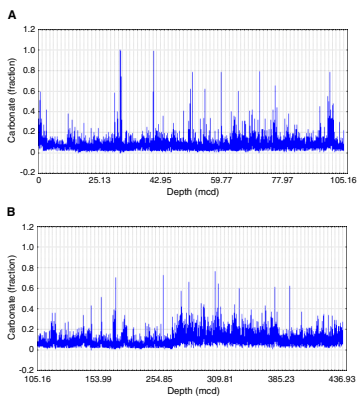

AFB5. Model-predicted carbonate fraction, Hole 1123A (laboratory and shipboard data) and Site 1123 (laboratory and shipboard data), p. 18.
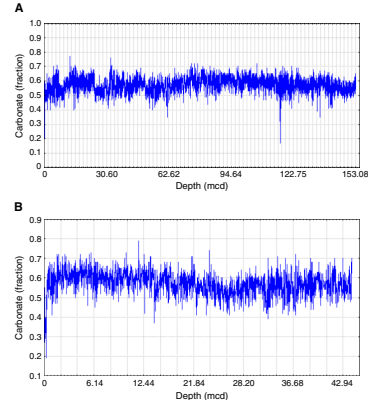

AFB6. Model-predicted carbonate fraction, Site 1124 (laboratory and shipboard data), p. 21.
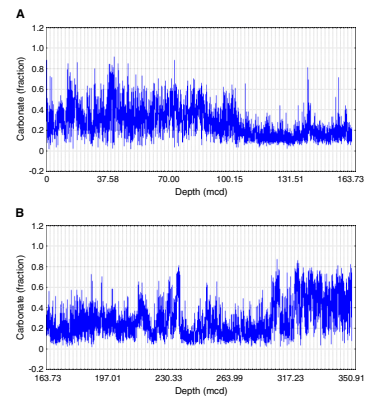

AFB7. Model-predicted carbonate fraction, Site 1125 (shipboard data), p. 23.

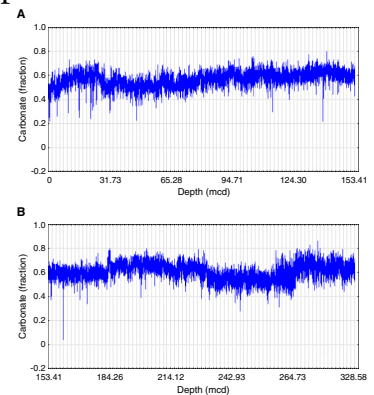




\section{APPENDIX C}

\section{Plots of Measured Carbonate Values vs. Predicted Values (Model Evaluation)}

The carbonate fraction prediction models were evaluated by comparing the plots of the Leg 198 predicted carbonate fraction values were to the measured carbonate fraction values. For results of the comparisons, see Figures AFC1, AFC2, AFC3, AFC4, AFC5, AFC6, and AFC7.

AFC1. Model-predicted carbonate fraction vs. measured carbonate fraction, Hole 1119C, p. 25.

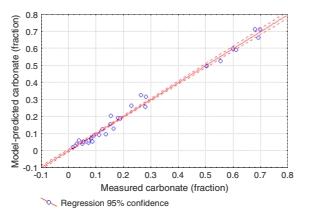

AFC2. Model-predicted carbonate fraction vs. measured carbonate fraction, Site 1120, p. 26.

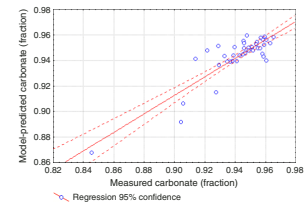

AFC3. Model-predicted carbonate fraction vs. measured carbonate fraction, Site 1121, p. 27.

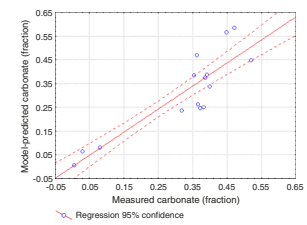

AFC4. Model-predicted carbonate fraction vs. measured carbonate fraction, Site 1122, p. 28.

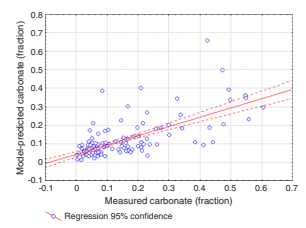

AFC5. Model-predicted carbonate fraction vs. measured carbonate fraction, Hole 1123A and Site 1123 , p. 29.
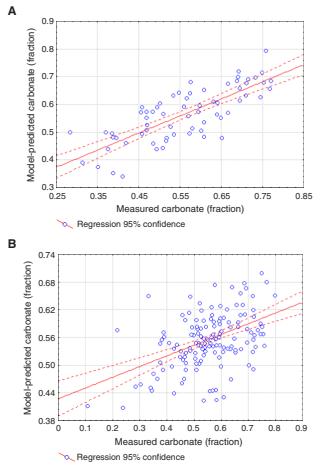

AFC6. Model-predicted carbonate fraction vs. measured carbonate fraction, Site 1124, p. 31.

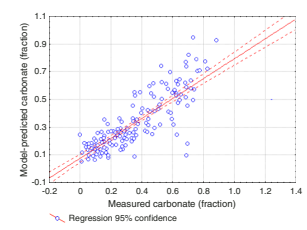

AFC7. Model-predicted carbonate fraction vs. measured carbonate fraction, Site 1125, p. 32.

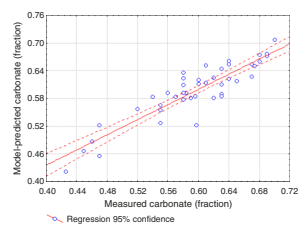




\section{APPENDIX D}

\section{Model Parameter Estimates}

Carbonate model parameters were estimated for Leg 198. Each of the following tables contains the carbonate model parameter estimates for the designated site or hole: Table ATD1 contains carbonate model parameter estimates for Site 1119C (laboratory reflectance data); Table ATD2 contains carbonate model parameter estimates for Site 1120; Table ATD3 contains carbonate model parameter estimates for Site 1121; Table ATD4 contains carbonate model parameter estimates for Site 1122; Table ATD5 contains carbonate model parameter estimates for Site 1123; Table ATD6 contains carbonate model parameter estimates for Hole 1123A (laboratory reflectance data); Table ATD7 contains carbonate model parameter estimates for Hole 1123A (shipboard reflectance data); Table ATD8 contains carbonate model parameter estimates for Site 1124; and Table ATD9 contains carbonate model parameter estimates for Site 1125.
ATD1. Carbonate model parameter estimates, Site 1119C (laboratory reflectance data), p. 42 .

ATD2. Carbonate model parameter estimates, Site 1120 , p. 43.

ATD3. Carbonate model parameter estimates, Site 1121, p. 44.

ATD4. Carbonate model parameter estimates, Site 1122, p. 45.

ATD5. Carbonate model parameter estimates, Site 1123, p. 46.

ATD6. Carbonate model parameter estimates, Hole 1123A (laboratory reflectance data), p. 47.

ATD7. Carbonate model parameter estimates, Hole 1123A (shipboard reflectance data), p. 48 .

ATD8. Carbonate model parameter estimates, Site 1124, p. 49.

ATD9. Carbonate model parameter estimates, Site 1125 , p. 50. 
Figure F1. Comparative display of the ranges of color reflectance data (all wavelengths combined for each hole). Dup $=$ shipboard data, prn $=$ laboratory data.

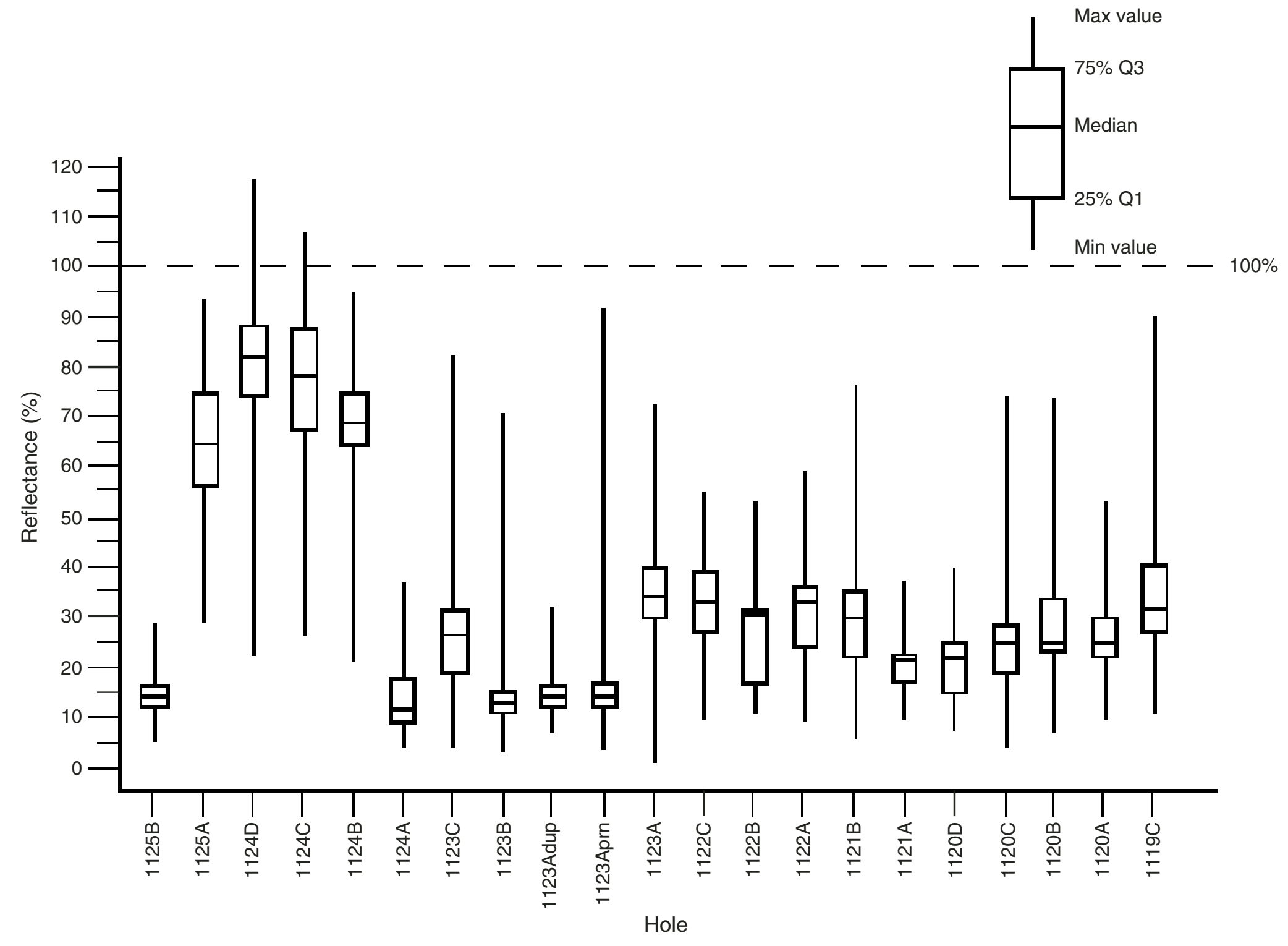


L.D. MILLWOOD ET AL.

Data Report: Color Reflectance as a Carbonate Proxy

Table T1. Measured carbonate summary statistics by site.

\begin{tabular}{clcccc}
\hline Site & $\begin{array}{c}100 \% \\
\text { maximum }\end{array}$ & Mean & $\begin{array}{c}0 \% \\
\text { minimum }\end{array}$ & Range & \multicolumn{2}{c}{ Carbonate } \\
\hline 1119 & 72.66 & 15.40861 & 0.48 & 72.18 & 74 \\
1120 & 98.73 & 94.59521 & 84.52 & 14.21 & 73 \\
1121 & 52.02 & 22.89152 & 0.38 & 51.64 & 33 \\
1122 & 76.41 & 13.25076 & 0.07 & 76.34 & 236 \\
1123 & 84.3 & 58.27108 & 10.93 & 73.37 & 912 \\
1124 & 88.28 & 34.98078 & 0 & 88.28 & 373 \\
1125 & 71 & 59.72153 & 23 & 48 & 144 \\
\hline
\end{tabular}

Note: $N=$ number of samples with measured carbonate (total). 
L.D. MILLWOOD ET AL.

Data Report: Color Reflectance as a Carbonate Proxy

Table T2. Carbonate prediction model statistics.

\begin{tabular}{|c|c|c|c|c|c|c|c|c|}
\hline Site/Hole & $\begin{array}{l}\text { Root mean } \\
\text { squared } \\
\text { error }\end{array}$ & $R^{2}$ & $\begin{array}{c}\text { Coefficient of } \\
\text { variation }\end{array}$ & $\begin{array}{c}\text { Reflectance } \\
N\end{array}$ & $\begin{array}{c}\text { Carbonate } \\
N\end{array}$ & $\begin{array}{c}\text { Variables } \\
\qquad N\end{array}$ & Model variables & $\begin{array}{l}\text { Data } \\
\text { source }\end{array}$ \\
\hline $1119 \mathrm{C}$ & 0.36437 & 0.9714 & -17.52781 & 2922 & 74 & 30 & all $30 \mathrm{fdv}$ & Lab \\
\hline 1120 & 0.19376 & 0.6609 & 6.78812 & 12184 & 73 & 5 & $\mathrm{r} 1, \mathrm{fd} 2, \mathrm{fd} 7, \mathrm{fd} 21, \mathrm{fd} 27$ & Ship \\
\hline 1121 & 0.48677 & 0.9606 & -24.46203 & 3602 & 33 & 5 & $\mathrm{r} 1, \mathrm{fd} 4, \mathrm{fd} 7, \mathrm{fd} 21, \mathrm{fd} 30$ & Ship \\
\hline 1122 & 0.97357 & 0.4473 & -41.56004 & 20967 & 236 & 31 & $\mathrm{r} 1, \mathrm{fd} 1-\mathrm{fd} 30$ & Ship \\
\hline 1123 & 0.45273 & 0.2895 & 157.96569 & 41844 & 912 & 31 & $\mathrm{r} 1, \mathrm{fd} 1-\mathrm{fd} 30$ & Ship \\
\hline $1123 \mathrm{~A}$ & 0.43911 & 0.6097 & 151.1393 & 833 & 280 & 30 & all $30 \mathrm{fdv}$ & Lab \\
\hline $1123 \mathrm{~A}$ & 0.49232 & 0.2191 & 212.54304 & 1868 & 337 & 30 & all $30 \mathrm{fdv}$ & Ship \\
\hline 1124 & 0.79314 & 0.6592 & -94.62303 & 27721 & 374 & 30 & all $30 \mathrm{fdv}$ & Ship \\
\hline 1125 & 0.20555 & 0.8166 & 50.9898 & 23412 & 144 & 30 & all $30 \mathrm{fdv}$ & Ship \\
\hline
\end{tabular}

Notes: $N=$ number of samples with measured reflectance, number of samples with measured carbonate (total), and number of variables used to construct models. $\mathrm{fd}=$ first derivative value for the interval $400-410 \mathrm{~nm}, \mathrm{fdv}=$ first derivative value, $\mathrm{r} 1$ = reflectance measurement for $400 \mathrm{~nm}$. 
L.D. MILLWOOD ET AL.

Data Report: Color Reflectance as a Carbonate Proxy

Figure AFB1. Model-predicted carbonate fraction for Hole 1119C (laboratory data).

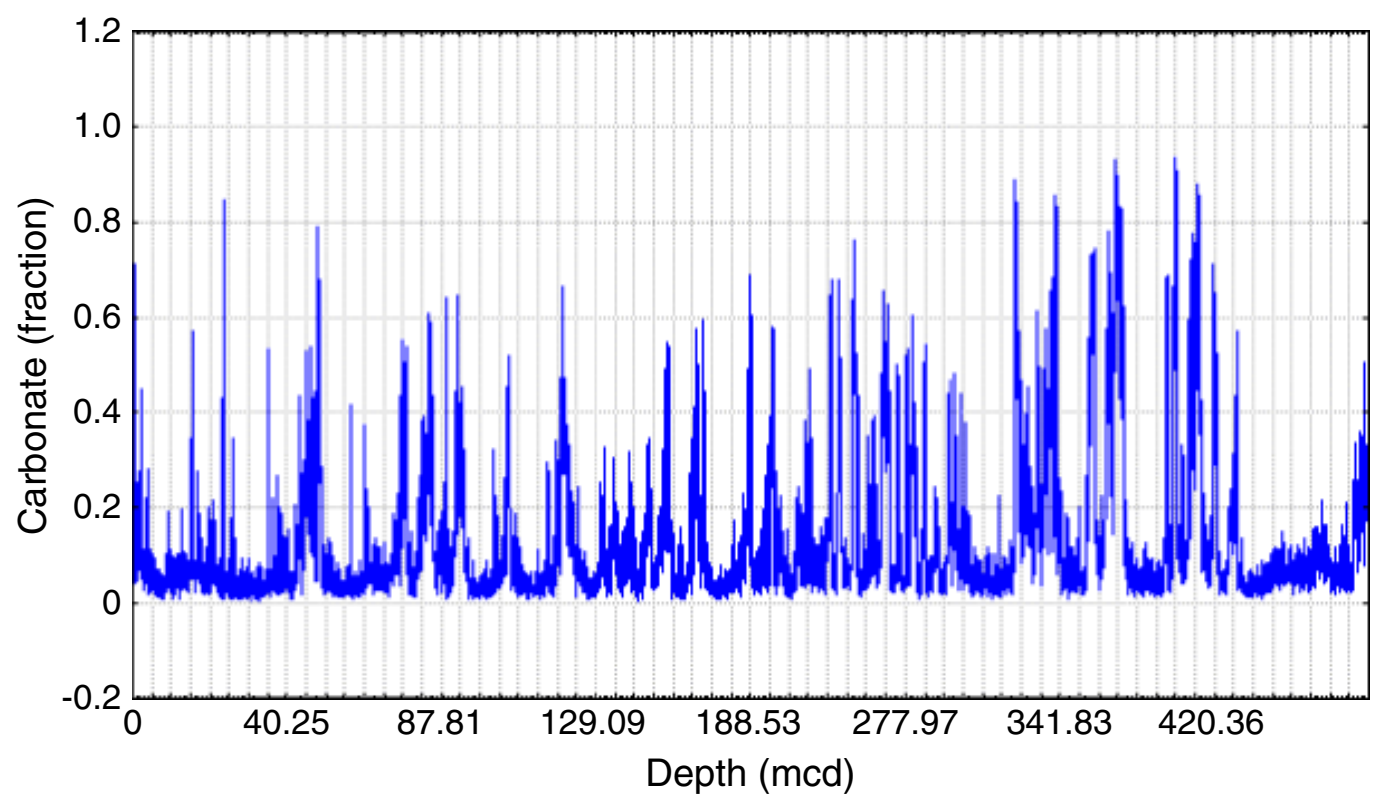


L.D. MILLWOOD ET AL.

Data Report: Color Reflectance as a Carbonate Proxy

Figure AFB2. A. Model-predicted carbonate fraction for Site 1120, cases 2-8001 (shipboard data). B. Modelpredicted carbonate fraction for Site 1120 (shipboard data), cases 8002-9054.

A

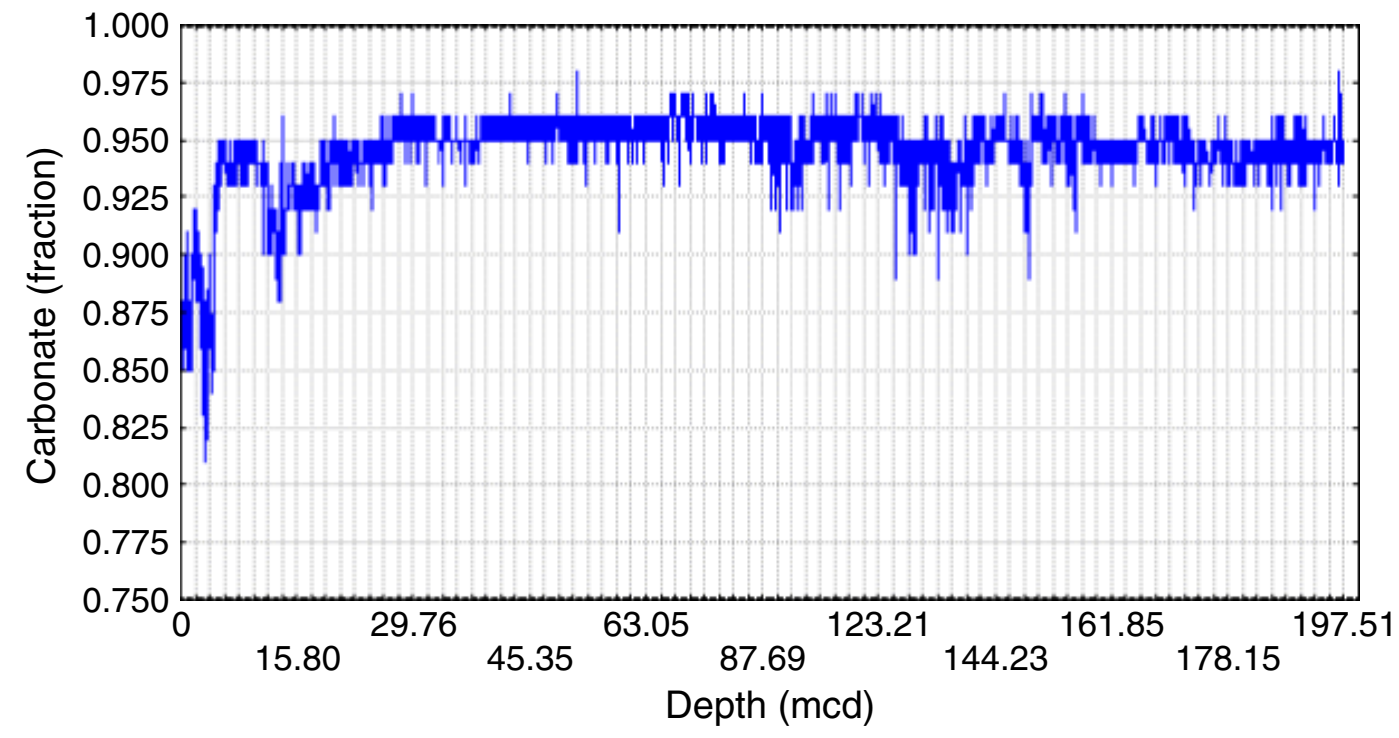

B

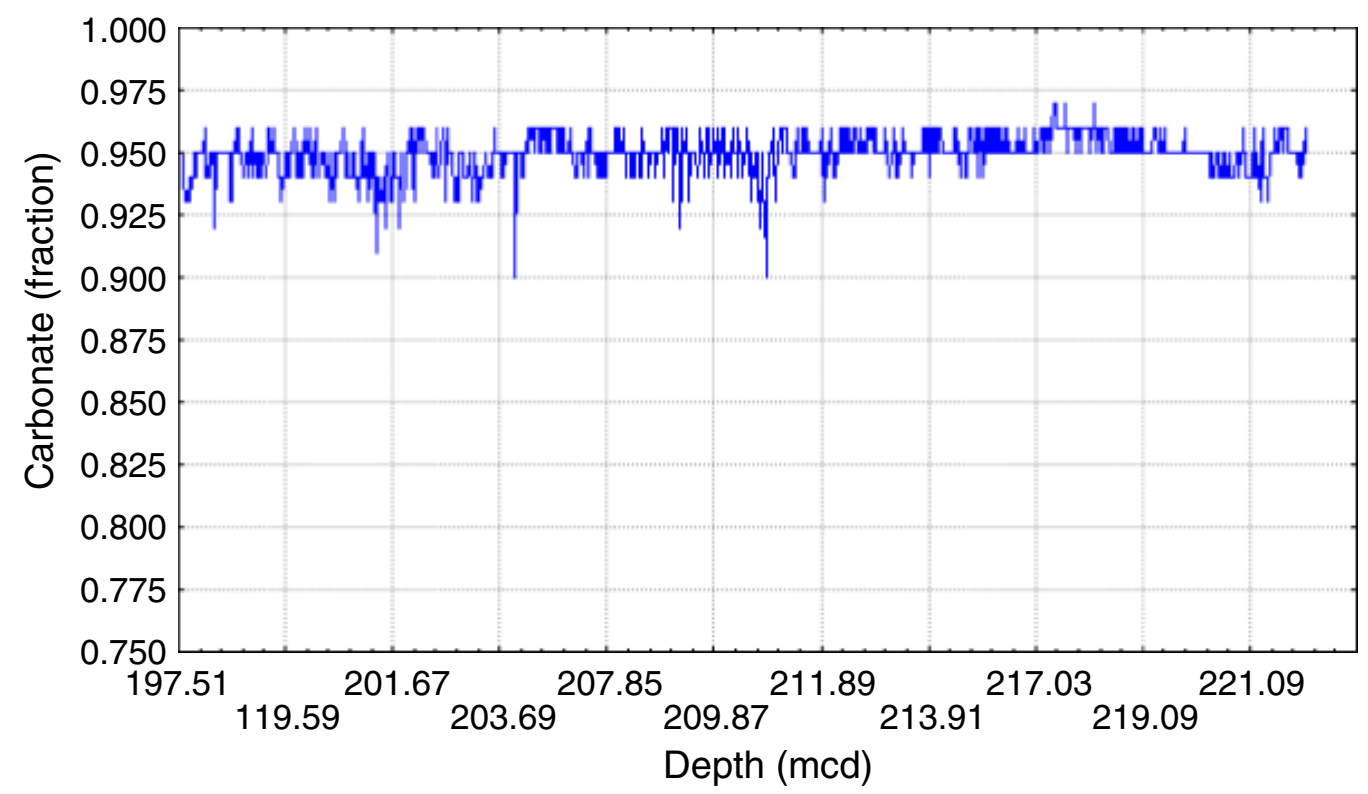


L.D. MILLWOOD ET AL.

Data Report: Color Reflectance as a Carbonate Proxy

Figure AFB3. Model-predicted carbonate fraction for Site 1121 (shipboard data), cases 2-8001.

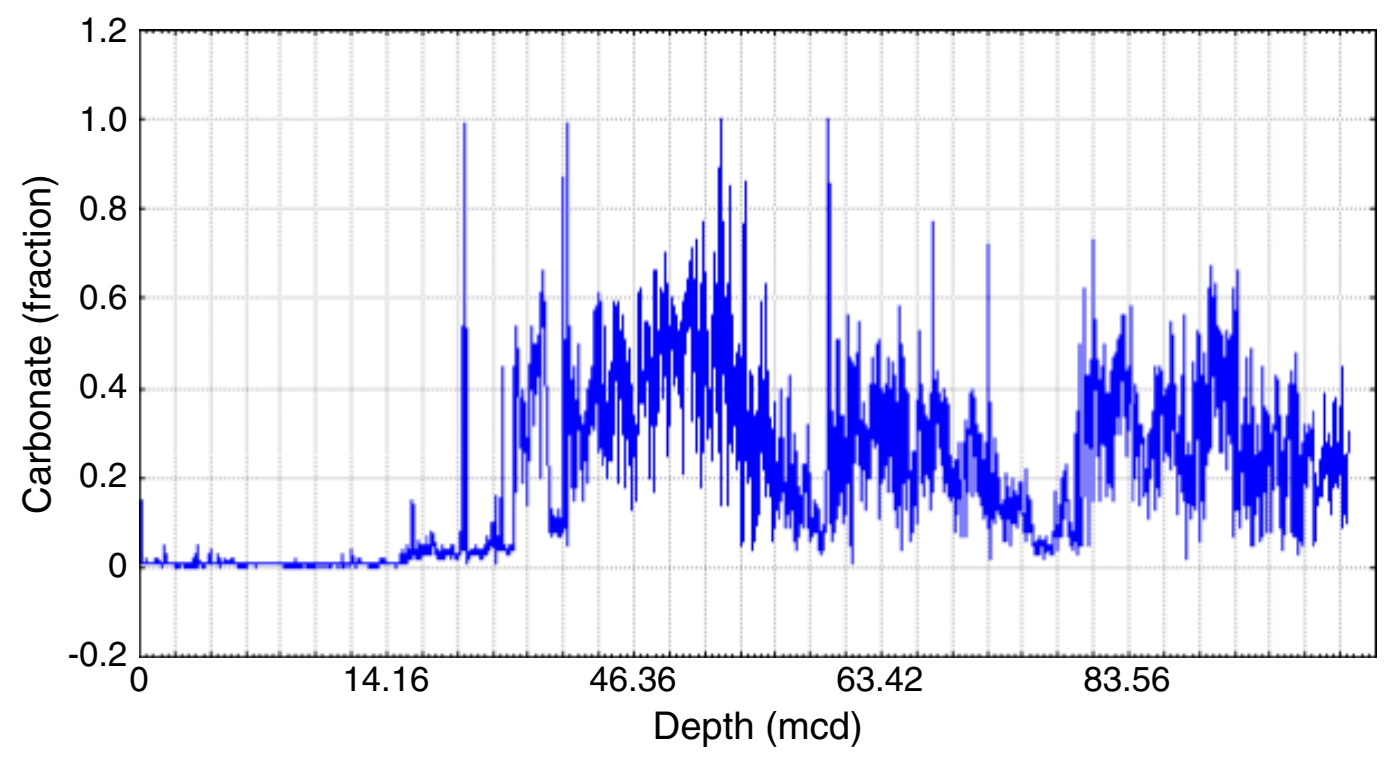


L.D. MILLWOOD ET AL.

Data Report: Color Reflectance as a Carbonate Proxy

Figure AFB4. A. Model-predicted carbonate fraction for Site 1122 (shipboard data), cases 2-8001. B. Modelpredicted carbonate fraction for Site 1122, cases 8002-16001. (Continued on next page.)

A

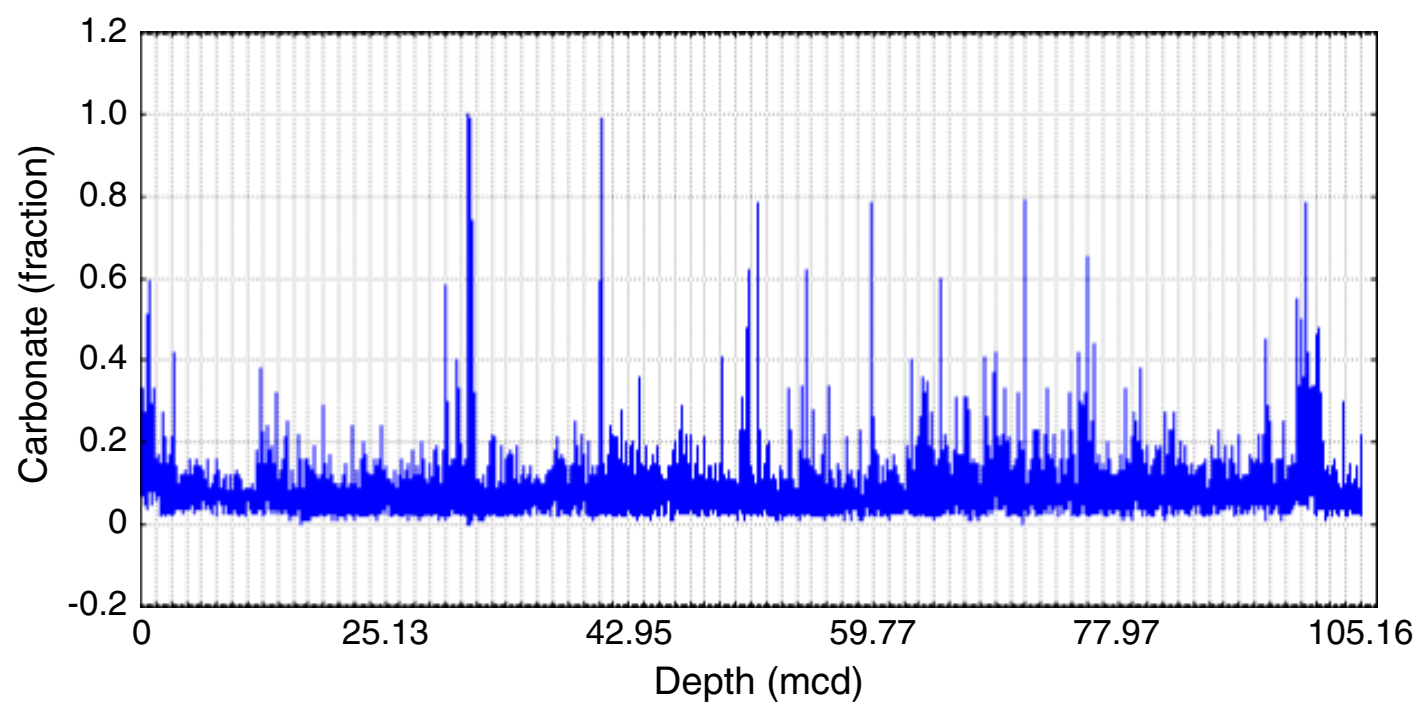

B

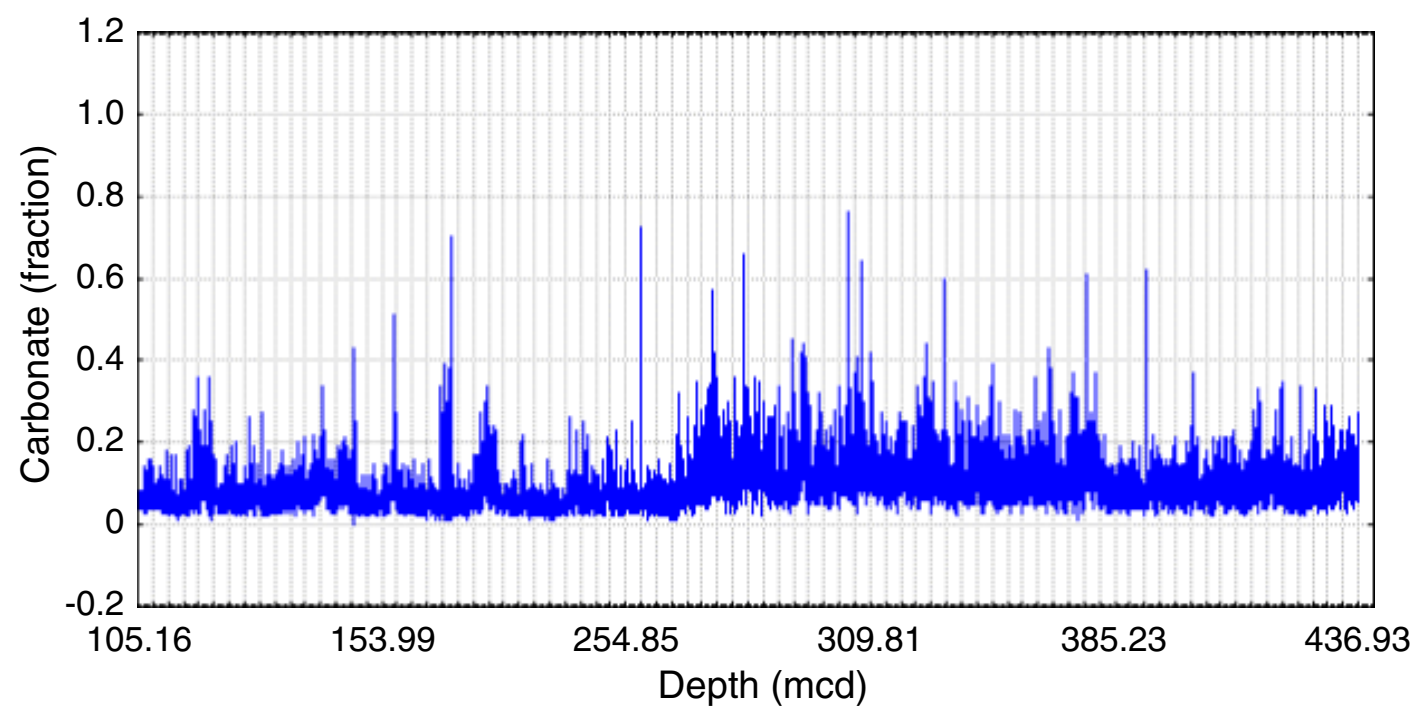


L.D. MILLWOOD ET AL.

Data Report: Color Reflectance as a Carbonate Proxy

Figure AFB4 (continued). C. Model-predicted carbonate fraction for Site 1122, cases 16002-19224.

C

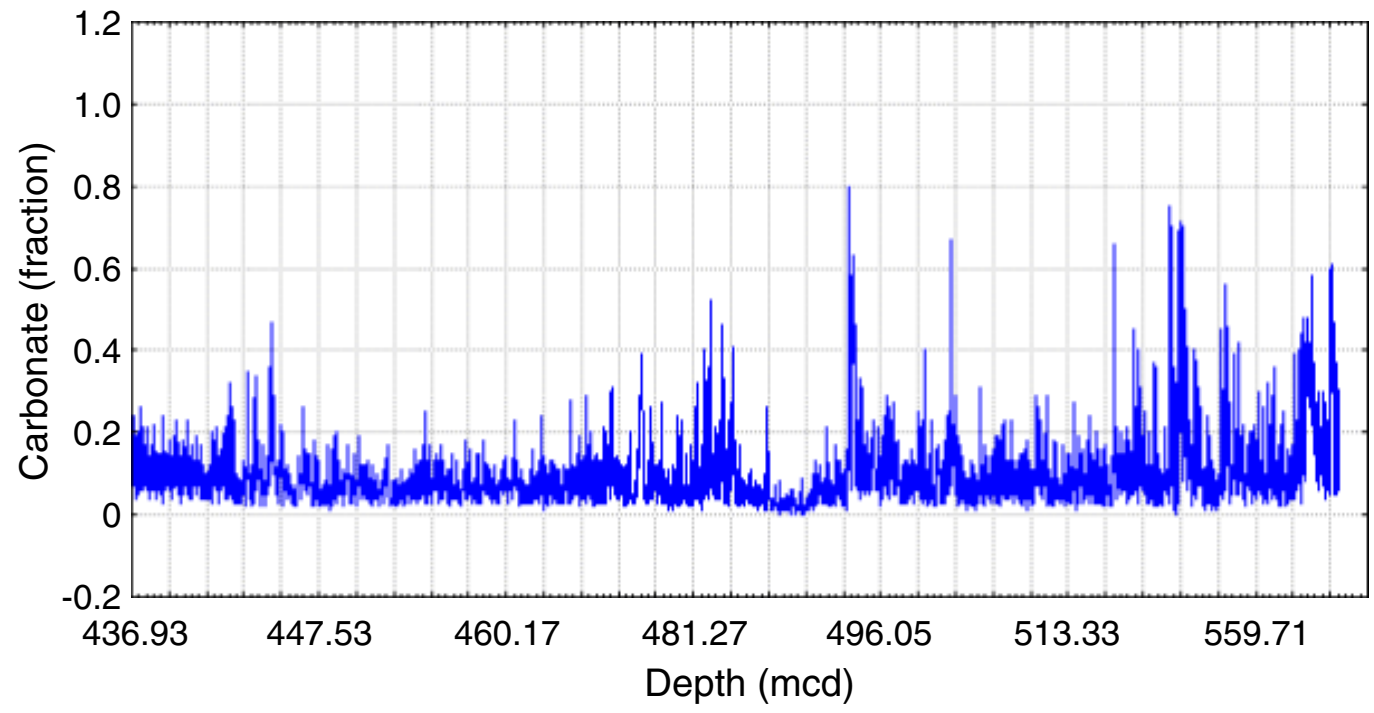


L.D. MILLWOOD ET AL.

Data Report: Color Reflectance as a Carbonate Proxy

Figure AFB5. A. Model-predicted carbonate fraction for Hole 1123A (laboratory data), cases 2-8001. B. Model-predicted carbonate fraction for Hole 1123A (shipboard data). Mcd = meters composite depth. (Continued on next two pages.)

A

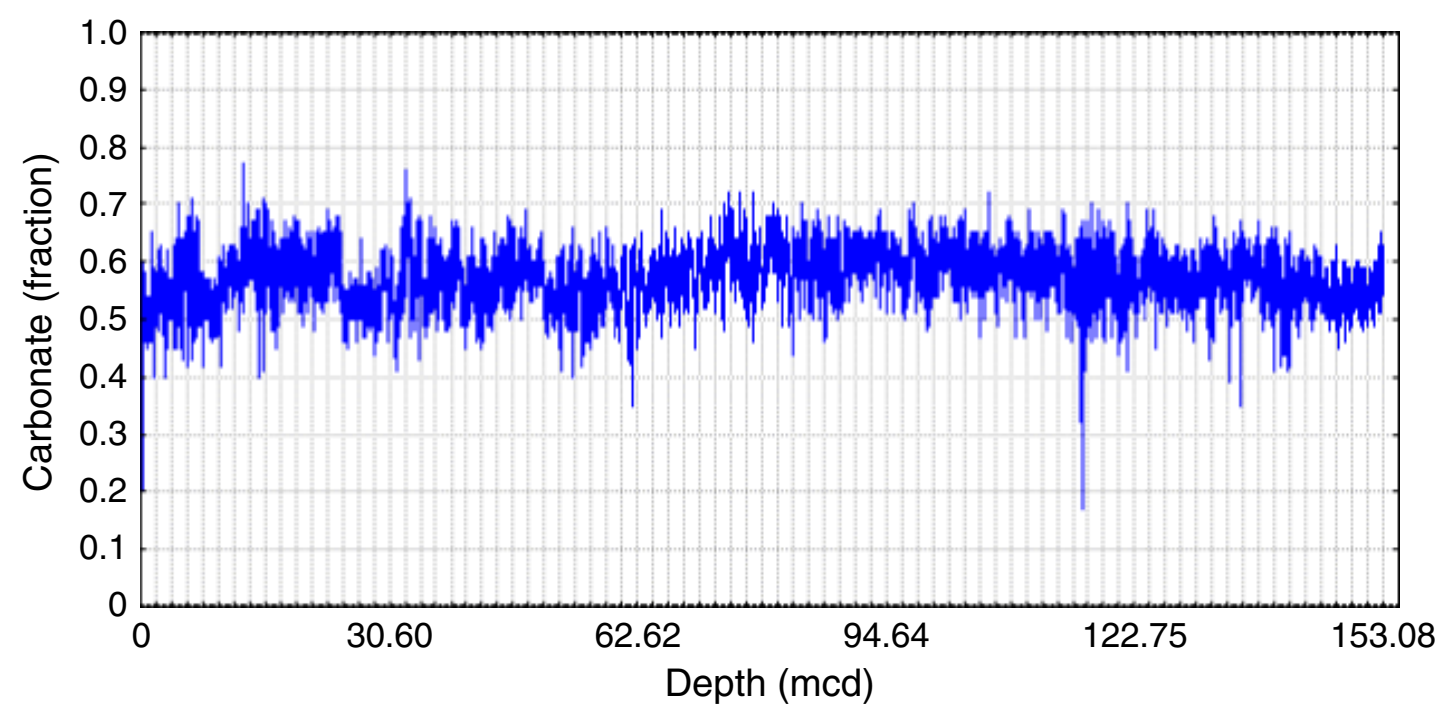

B

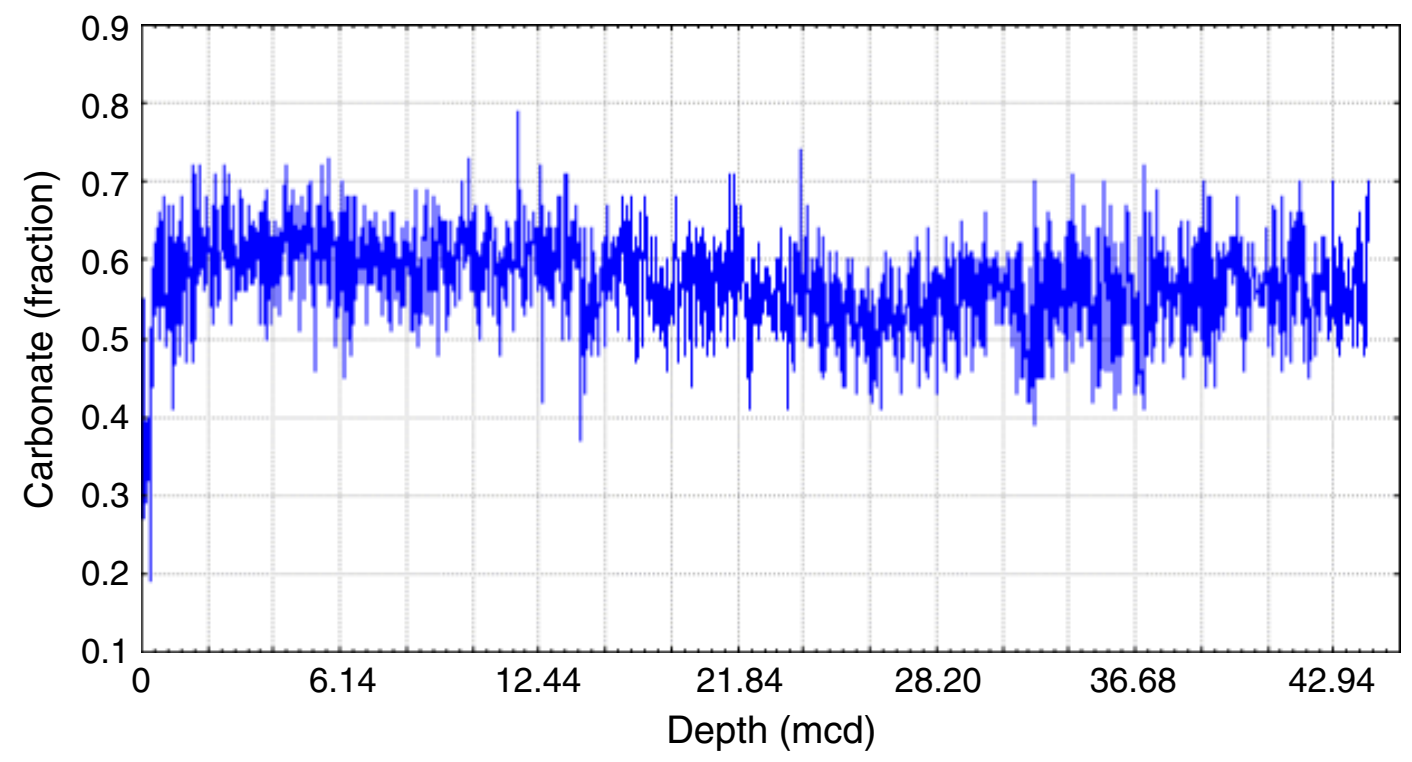


L.D. MILLWOOD ET AL.

Data Report: Color Reflectance as a Carbonate Proxy

Figure AFB5 (continued). C. Model-predicted carbonate fraction for Site 1123 (shipboard data), cases 28001. D. Model-predicted carbonate fraction for Site 1123 (shipboard data), cases 8002-16001. (Continued on next page.)

C

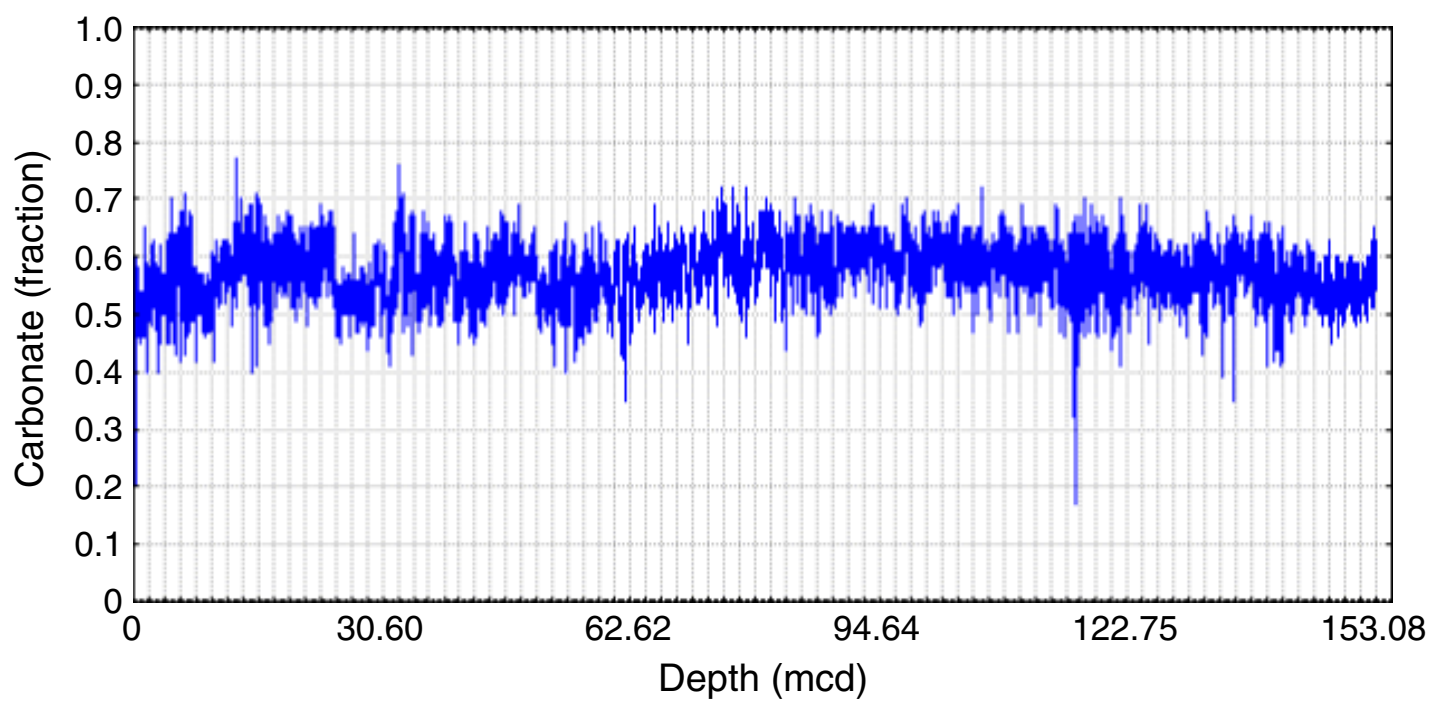

D

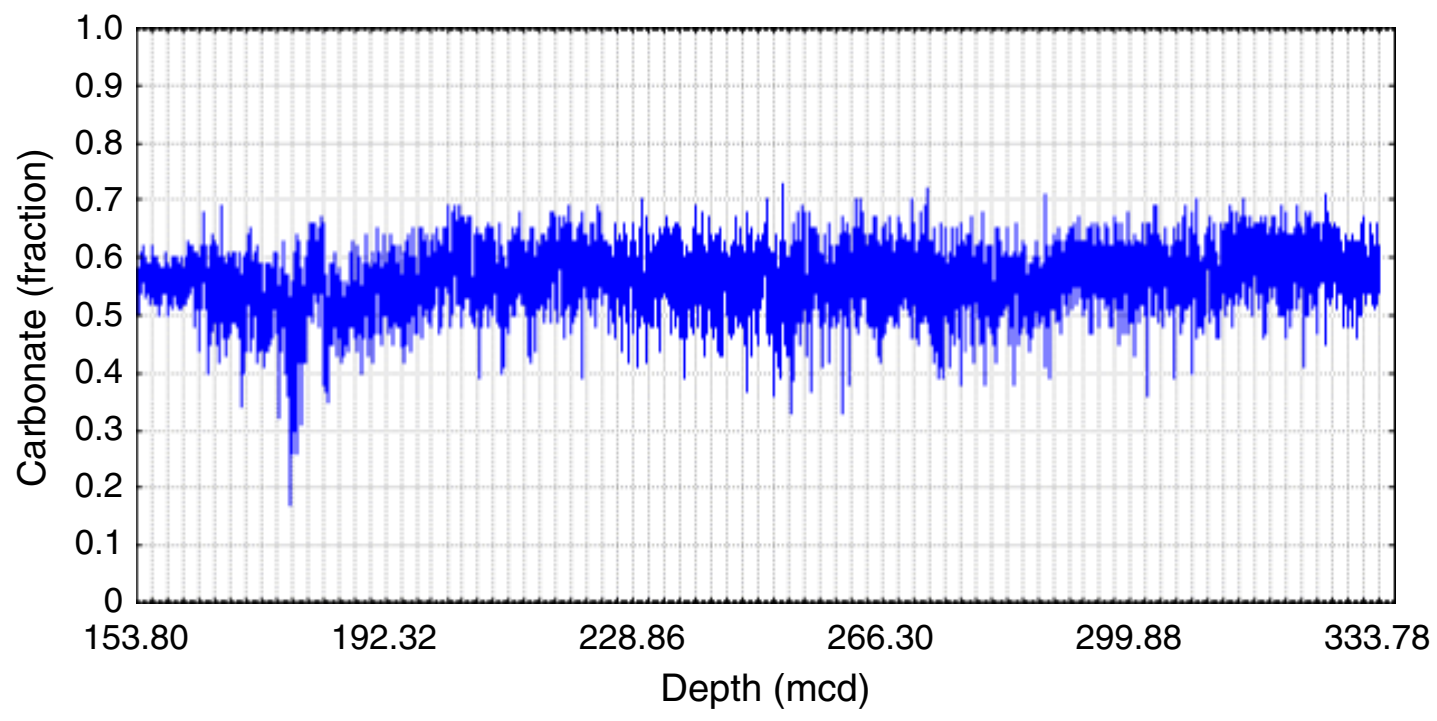


L.D. MILLWOOD ET AL.

Data Report: Color Reflectance as a Carbonate Proxy

Figure AFB5 (continued). E. Model-predicted carbonate fraction for Site 1123 (shipboard data), cases 16002-24001.

E

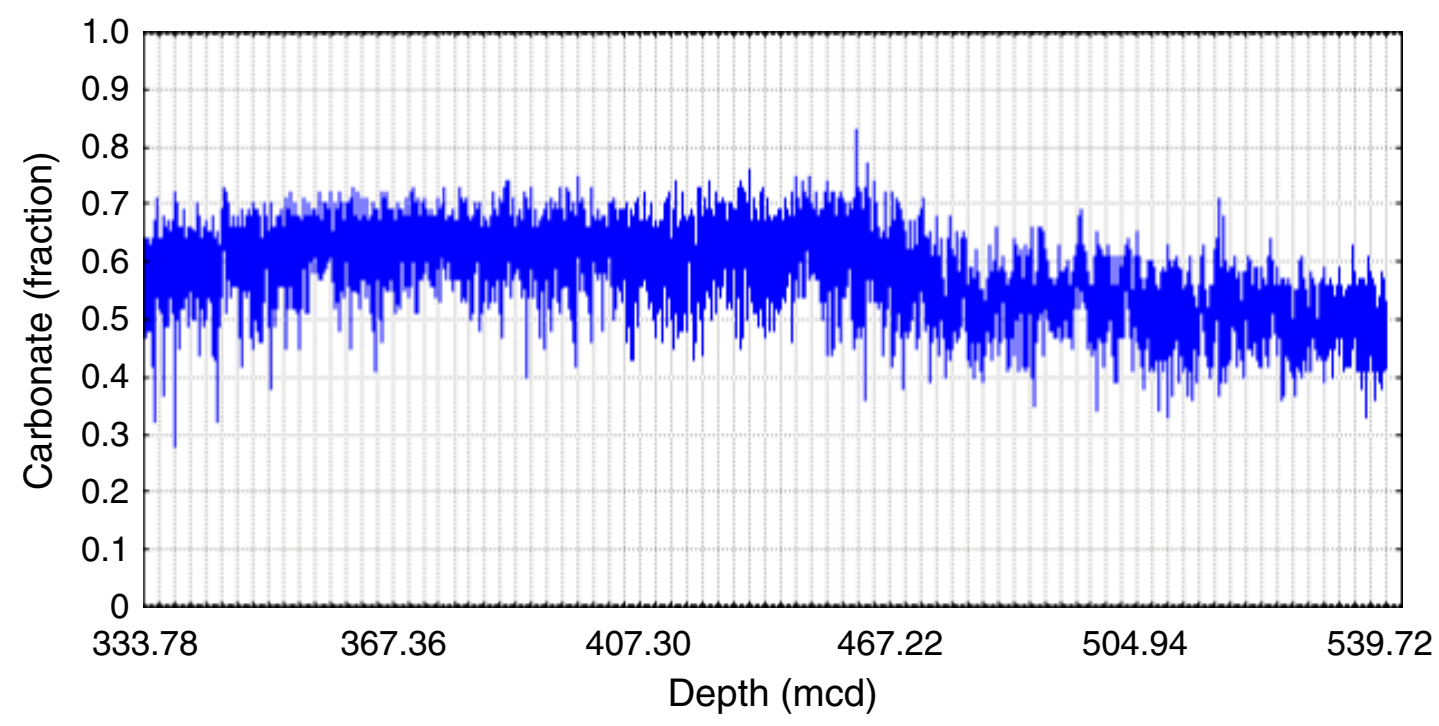


L.D. MILLWOOD ET AL.

Data Report: Color Reflectance as a Carbonate Proxy

Figure AFB6. A. Model-predicted carbonate fraction for Site 1124 (shipboard data), cases 2-8001. B. Modelpredicted carbonate fraction for Site 1124 (shipboard data), cases 8002-16001. (Continued on next page.)

A

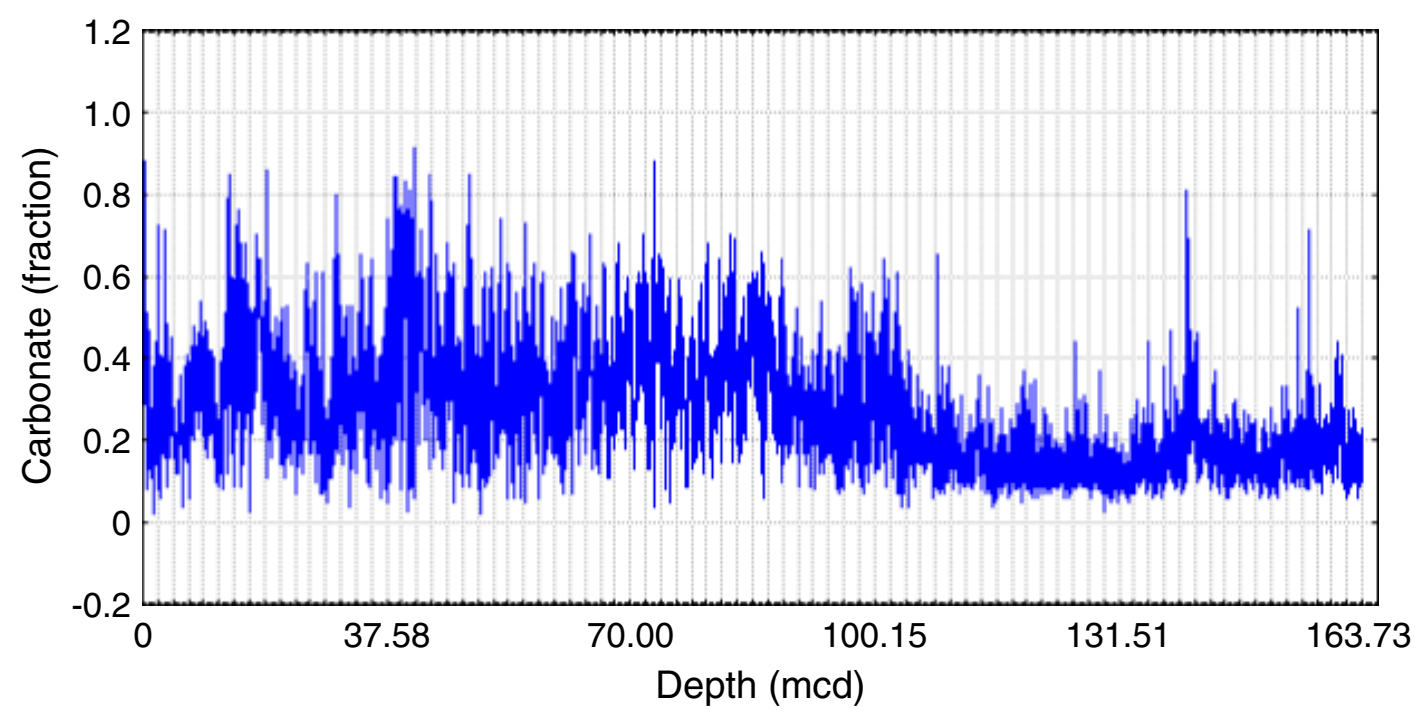

B

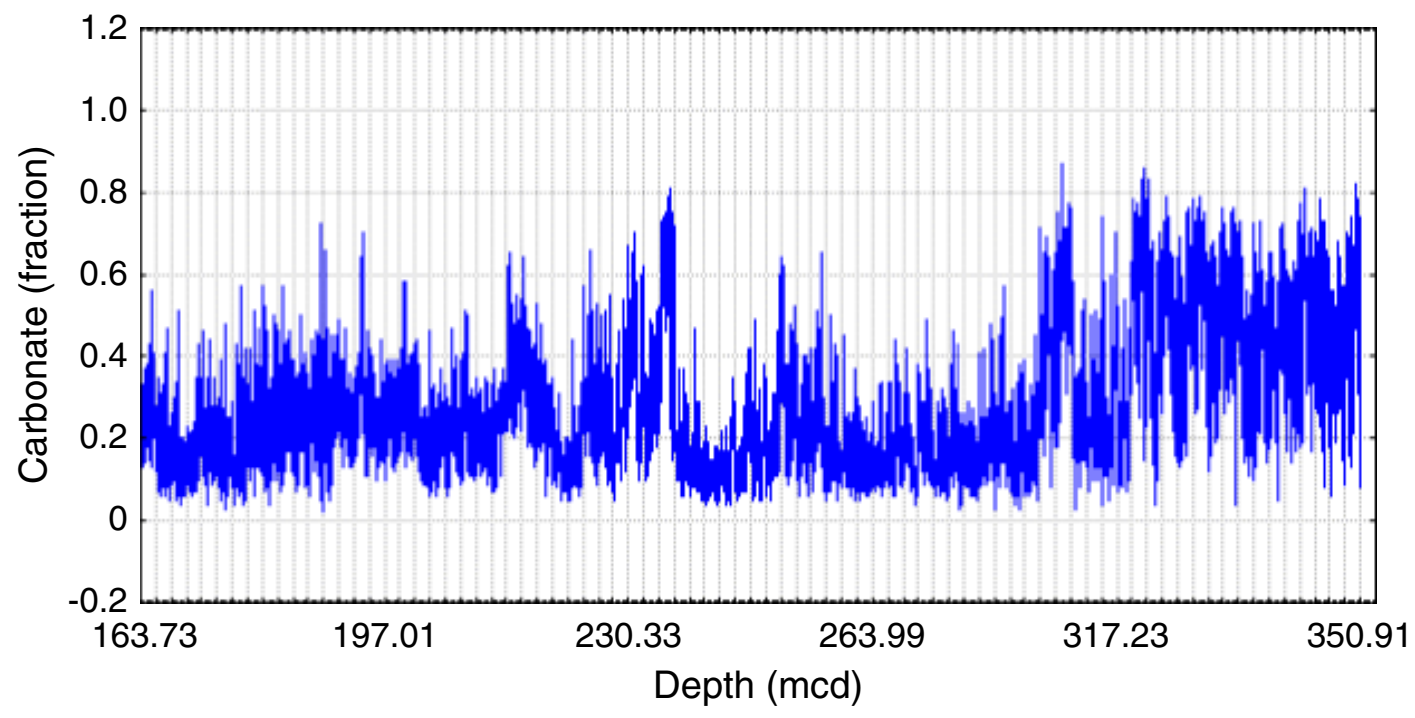


L.D. MILLWOOD ET AL.

Data Report: Color Reflectance as a Carbonate Proxy

Figure AFB6 (continued). C. Model-predicted carbonate fraction for Site 1124 (shipboard data), cases 16002-22055.

C

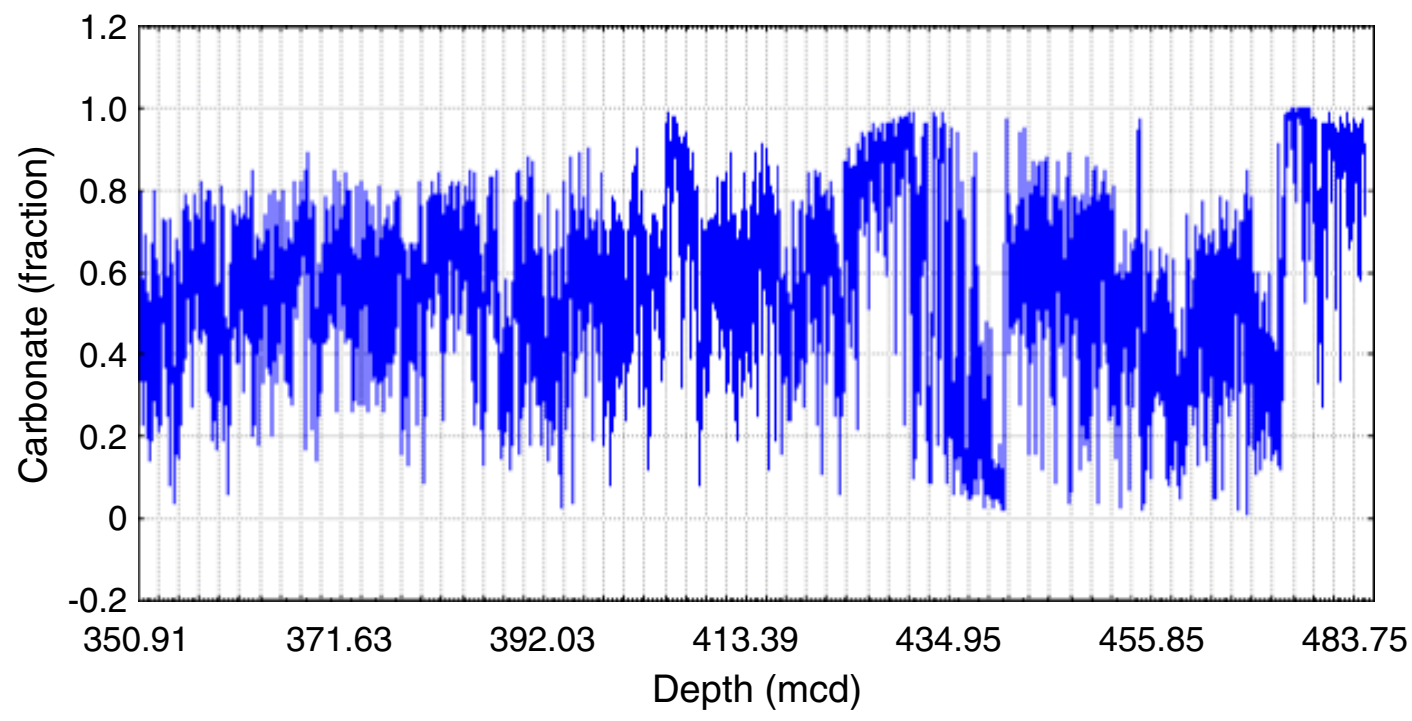


L.D. MILLWOOD ET AL.

Data Report: Color Reflectance as a Carbonate Proxy

Figure AFB7. A. Model-predicted carbonate fraction for Site 1125 (shipboard data), cases 2-8001. B. Modelpredicted carbonate fraction for Site 1125 (shipboard data), cases 8002-16001. (Continued on next page.)

A

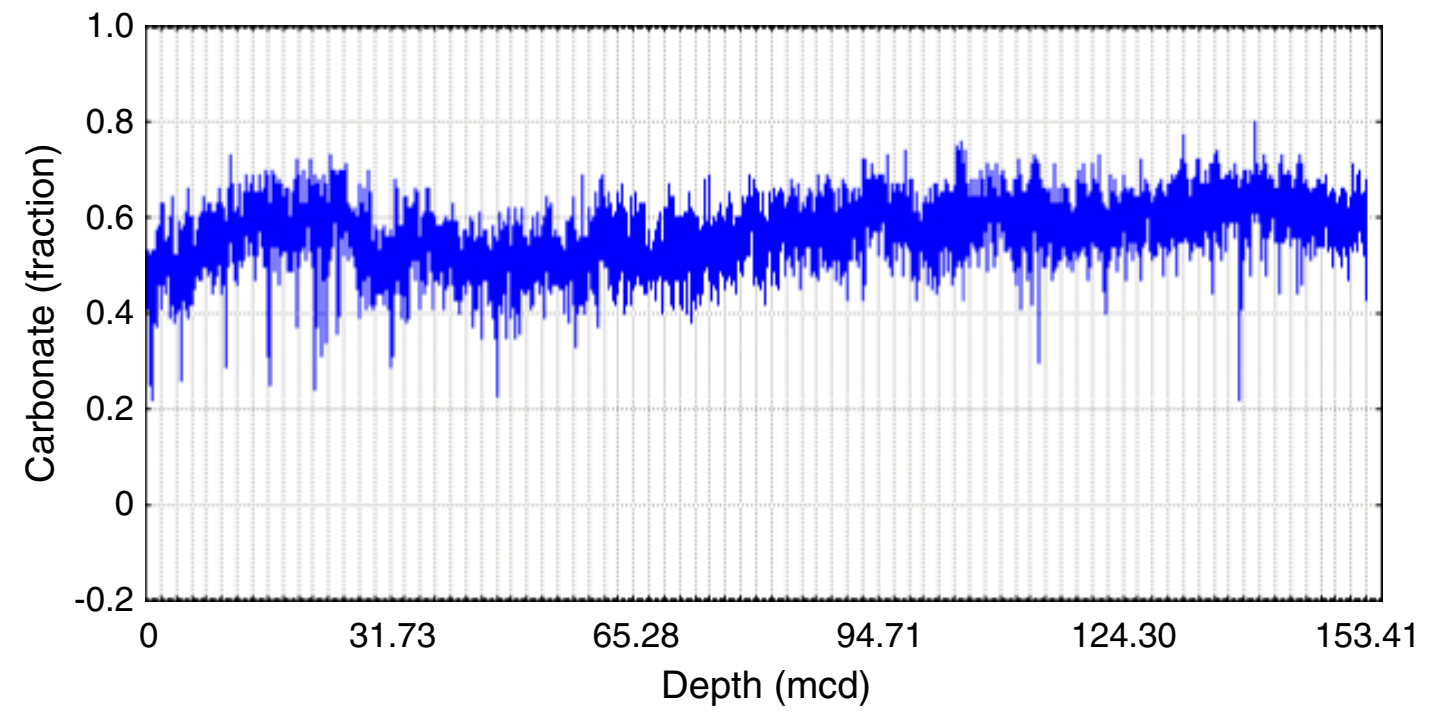

B

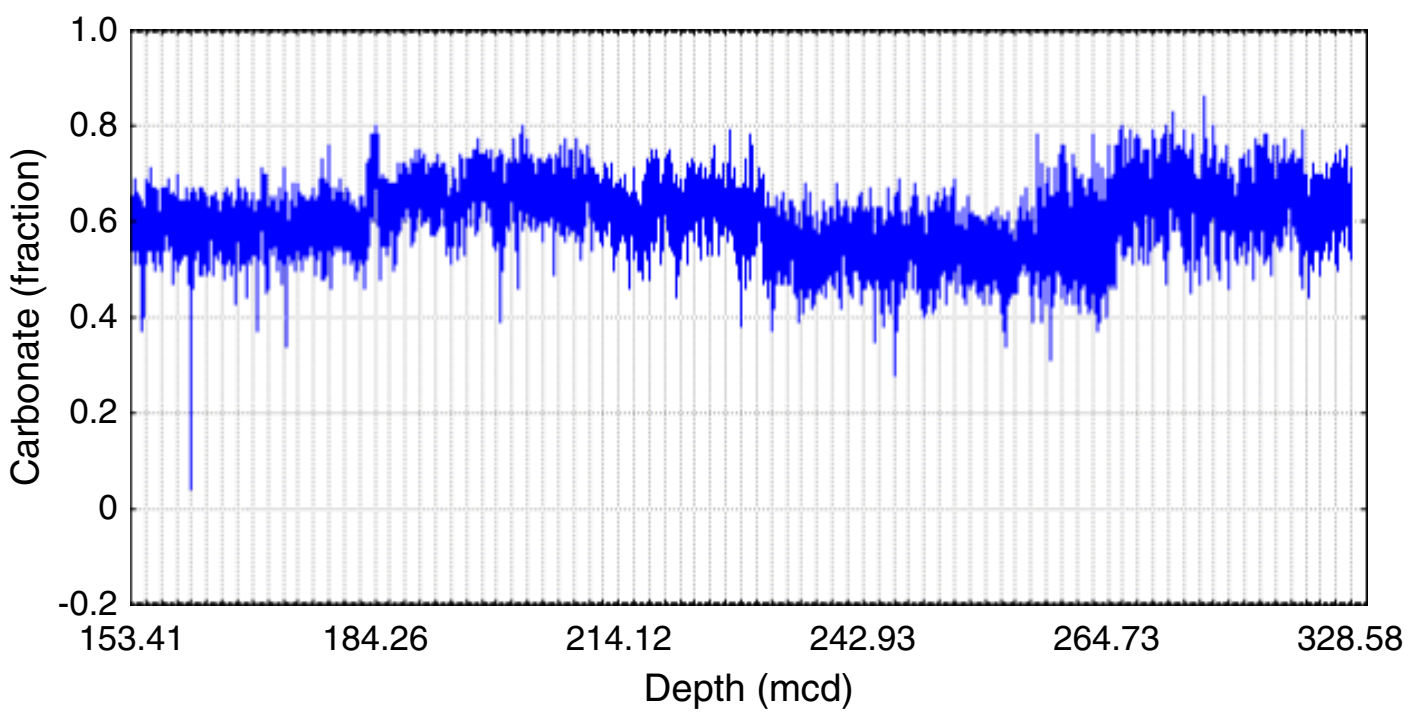


L.D. MILLWOOD ET AL.

Data Report: Color Reflectance as a Carbonate Proxy

Figure AFB7 (continued). C. Model-predicted carbonate fraction for Site 1125 (shipboard data), cases 16002-21723.

C

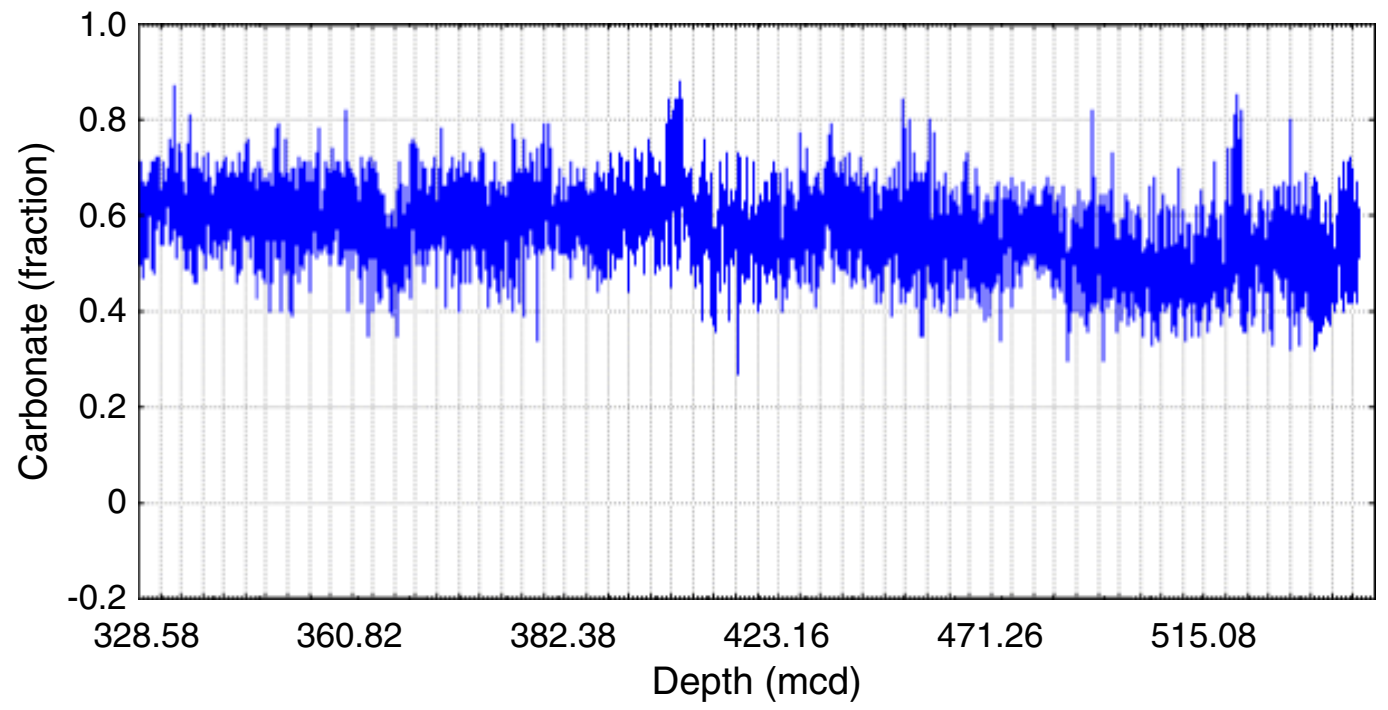


L.D. MILLWOOD ET AL.

Data Report: Color Reflectance as a Carbonate Proxy

Figure AFC1. Model-predicted carbonate fraction vs. measured carbonate fraction for Hole 1119C. Correlation: $r=0.99430$.

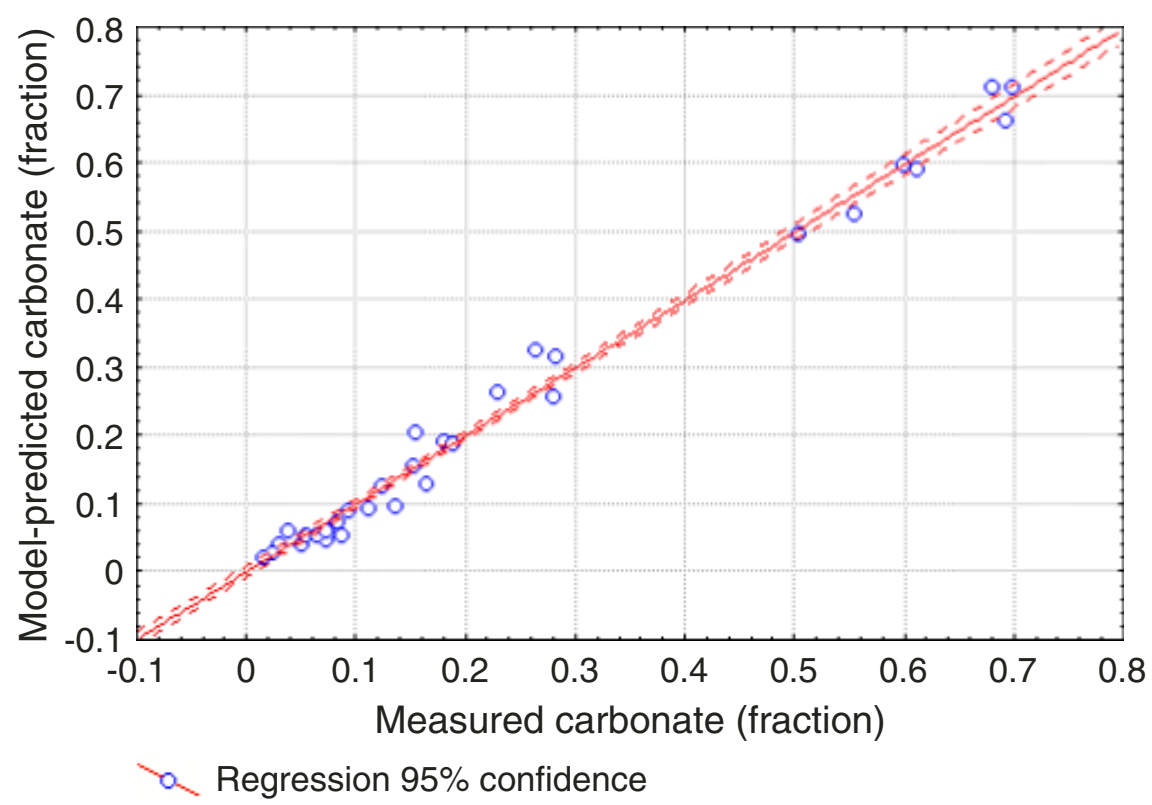


L.D. MILLWOOD ET AL.

Data Report: Color Reflectance as a Carbonate Proxy

Figure AFC2. Model-predicted carbonate fraction vs. measured carbonate fraction for Site 1120. Correlation: $r=0.87100$.

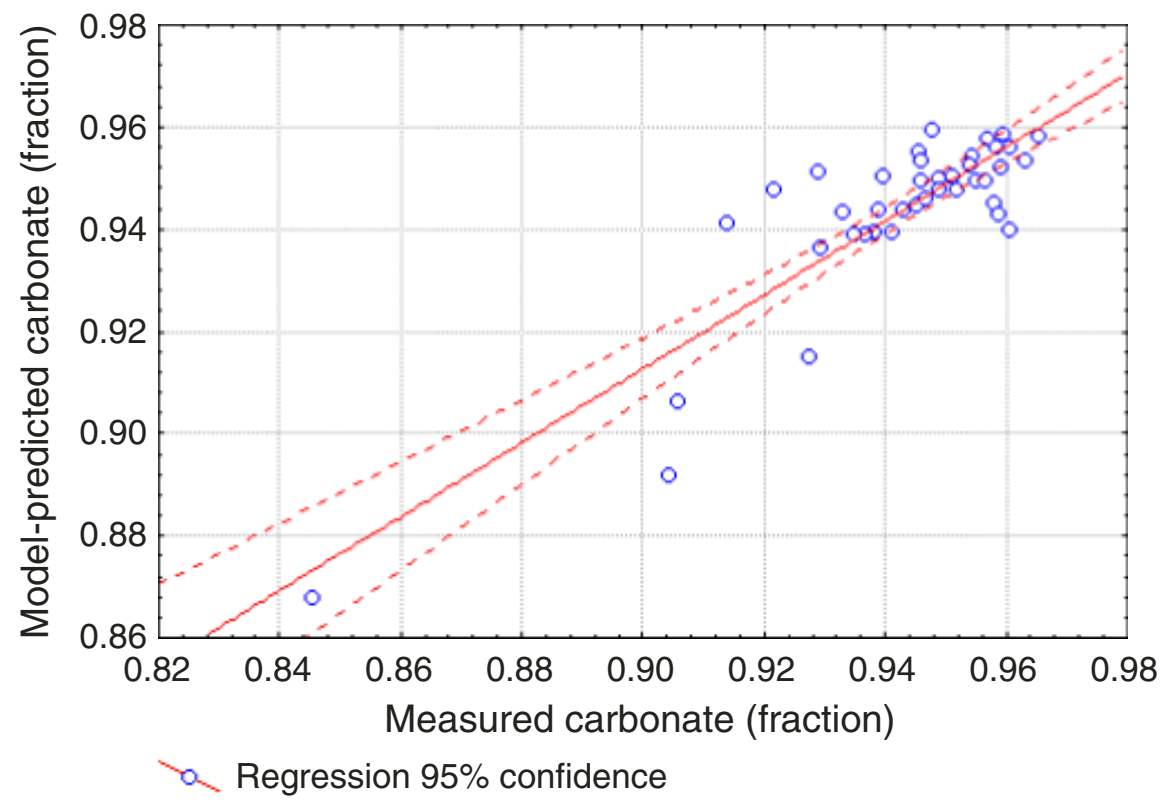


L.D. MILLWOOD ET AL.

Data Report: Color Reflectance as a Carbonate Proxy

Figure AFC3. Model-predicted carbonate fraction vs. measured carbonate fraction for Site 1121. Correlation: $r=0.93523$.

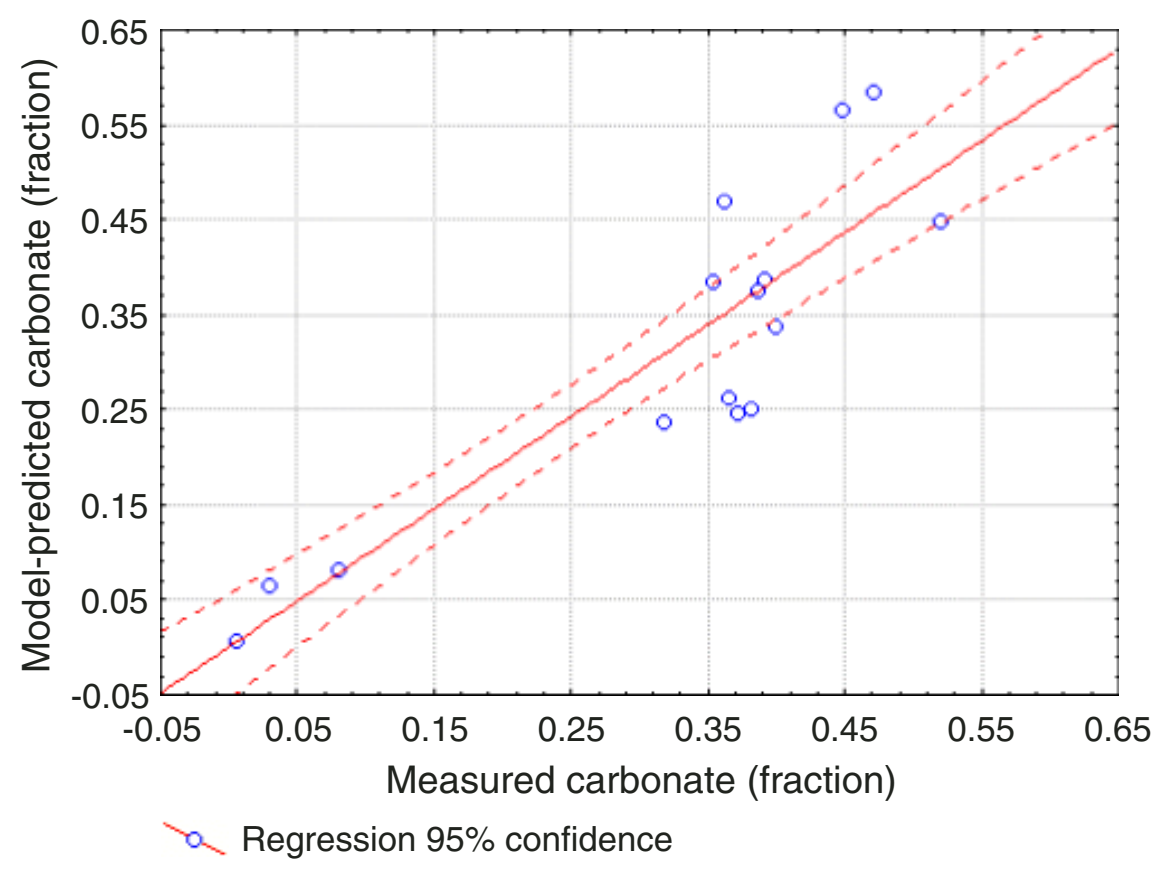


L.D. MILLWOOD ET AL.

Data Report: Color Reflectance as a Carbonate Proxy

Figure AFC4. Model-predicted carbonate fraction vs. measured carbonate fraction for Site 1122. Correlation: $r=0.68955$.

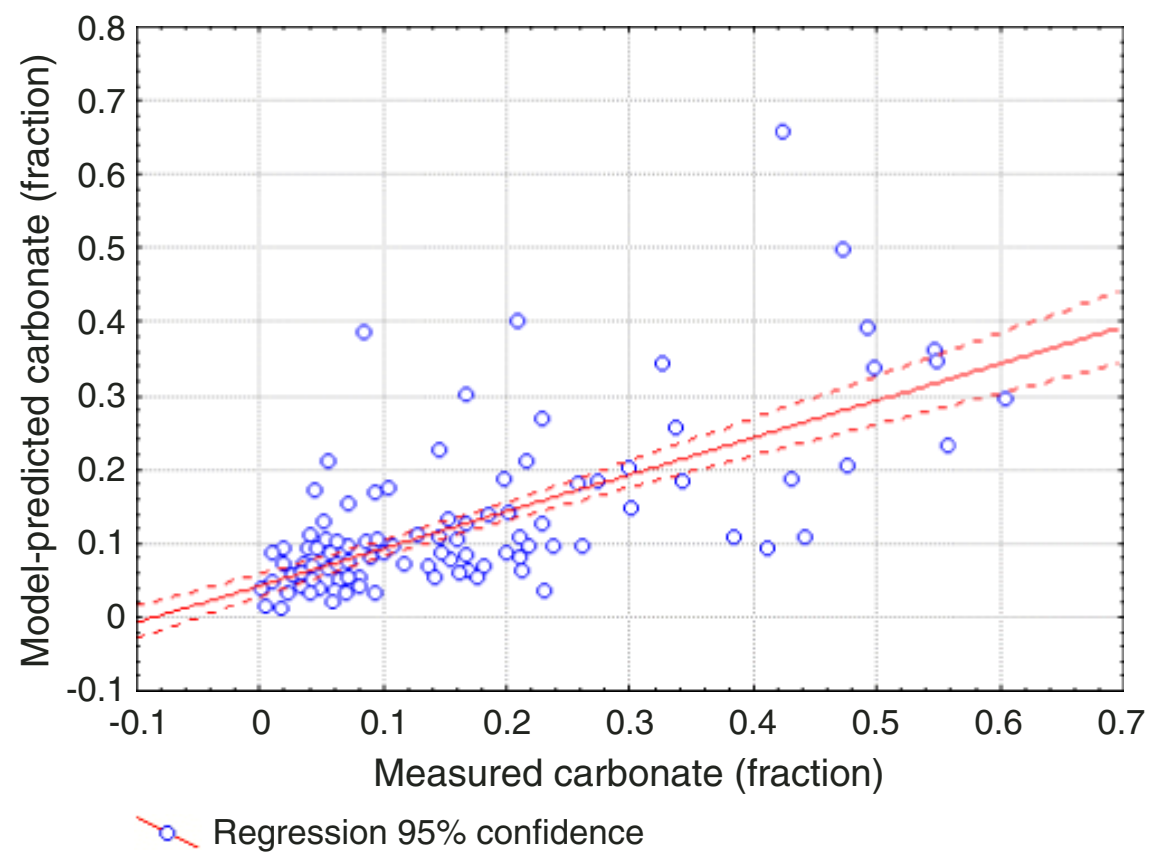


L.D. MILLWOOD ET AL.

Data Report: Color Reflectance as a Carbonate Proxy

Figure AFC5. A. Model-predicted carbonate fraction vs. measured carbonate fraction for Hole 1123A (laboratory data). Correlation: $r=0.78046$. B. Model-predicted carbonate fraction vs. measured carbonate fraction for Hole 1123A. $r=0.46702$. (Continued on next page.)

A

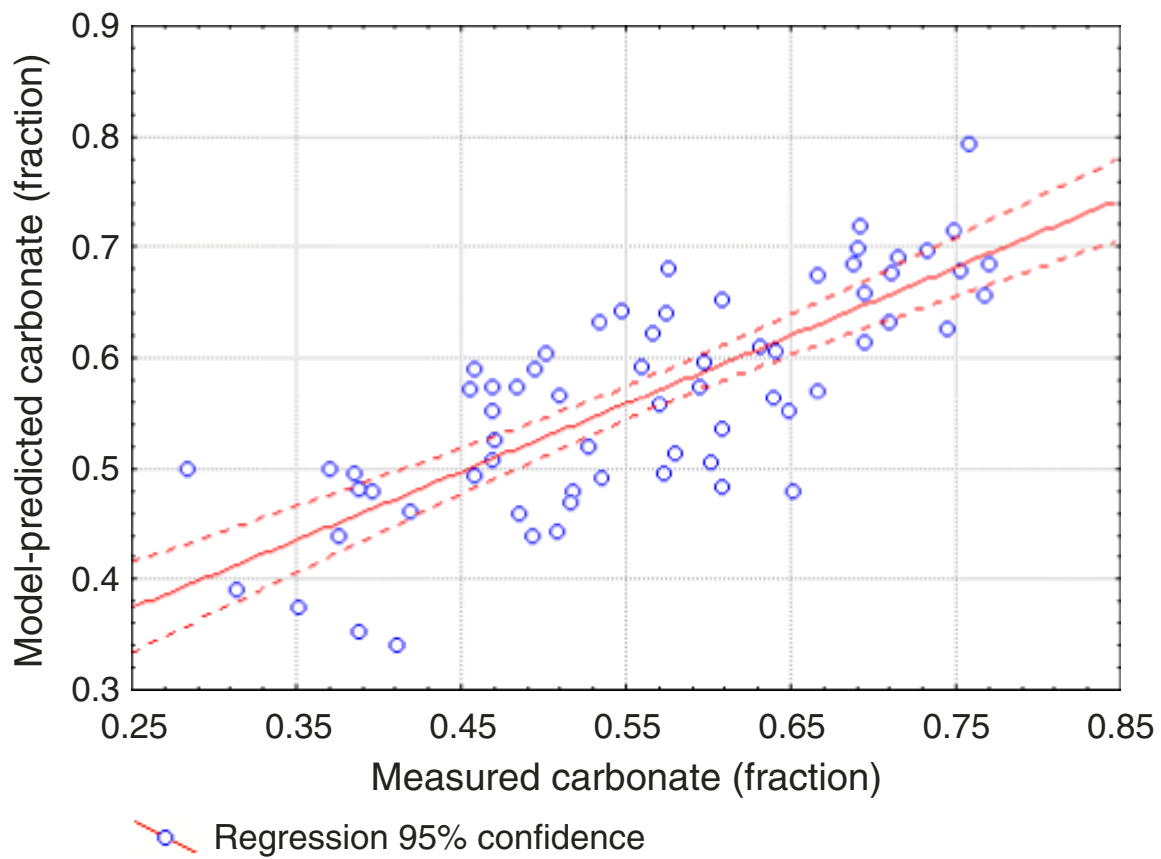

B

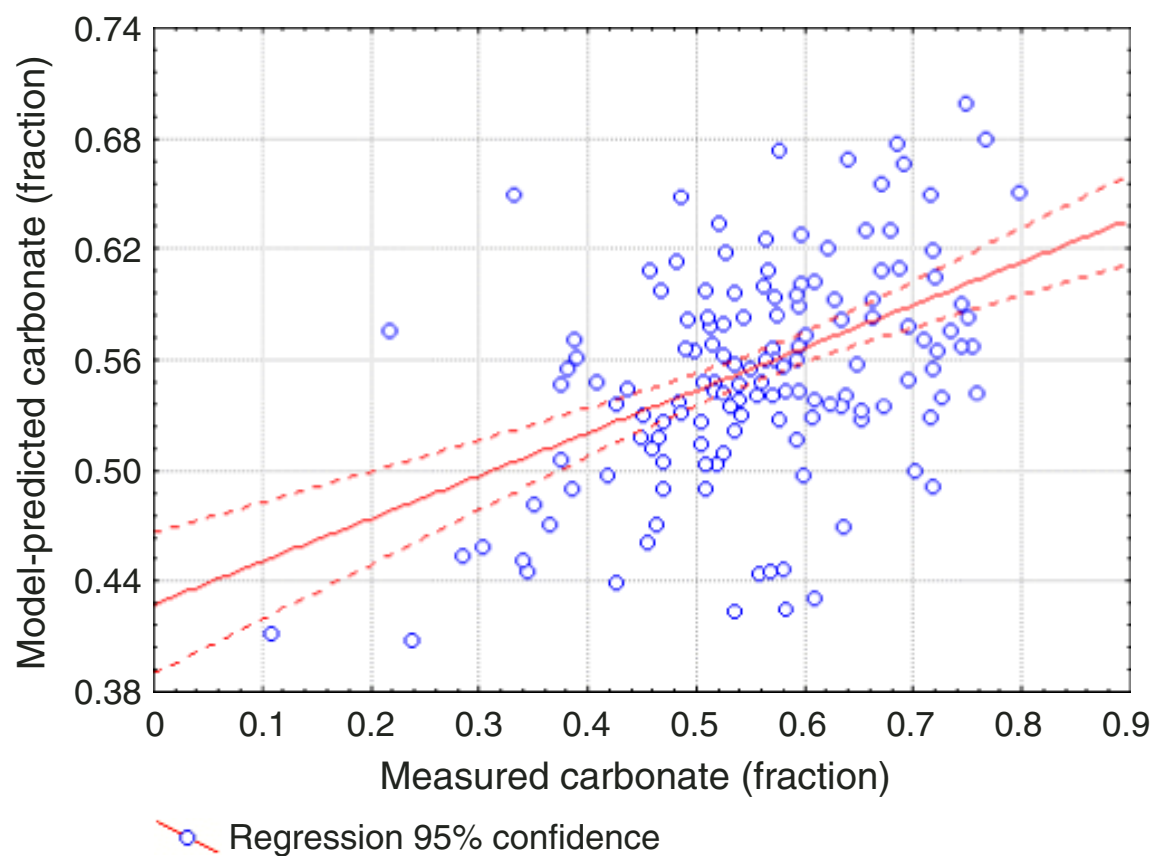


L.D. MILLWOOD ET AL.

Data Report: Color Reflectance as a Carbonate Proxy

Figure AFC5 (continued). C. Model-predicted carbonate fraction vs. measured carbonate fraction for Site 1123. Correlation: $r=0.53853$.

C

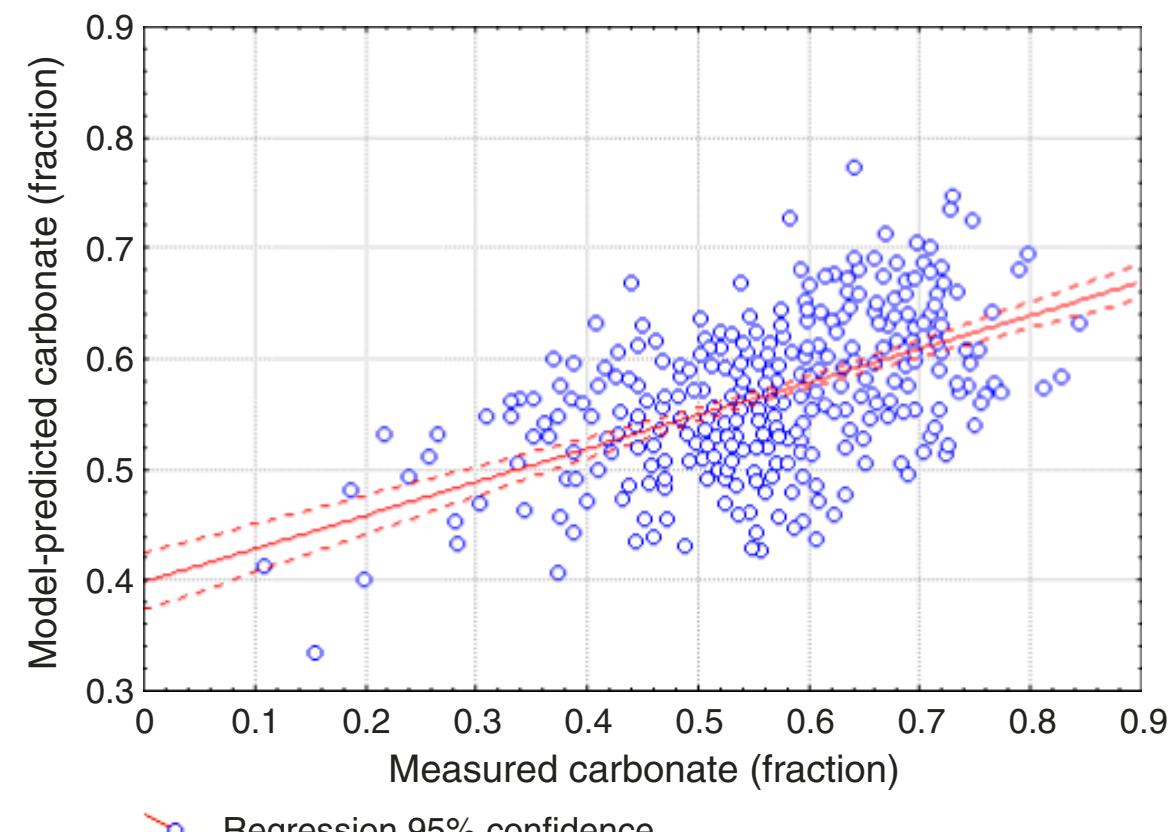

a. Regression $95 \%$ confidence 
L.D. MILLWOOD ET AL.

Data Report: Color Reflectance as a Carbonate Proxy

Figure AFC6. Model-predicted carbonate fraction vs. measured carbonate fraction for Site 1124. Correlation: $r=0.83085$.

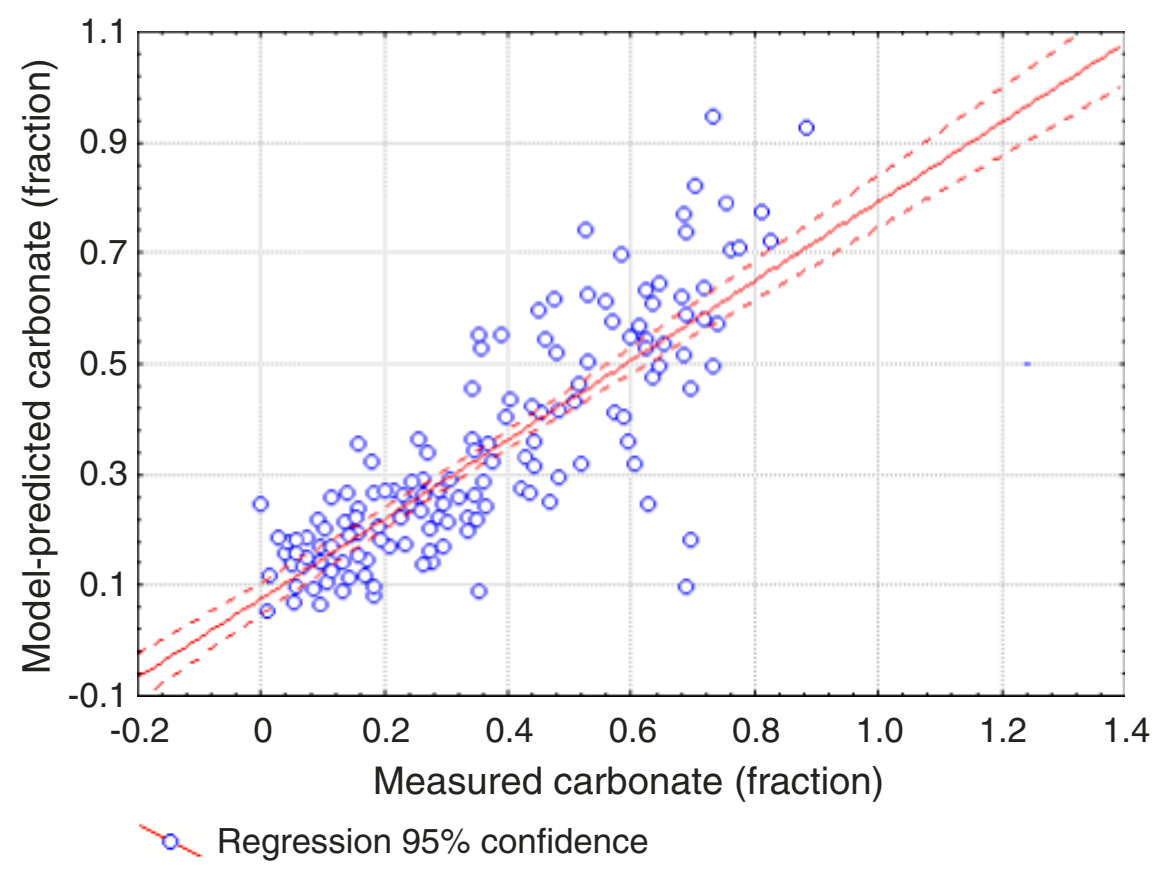


L.D. MILLWOOD ET AL.

Data Report: Color Reflectance as a Carbonate Proxy

Figure AFC7. Model-predicted carbonate fraction vs. measured carbonate fraction for Site 1125. Correlation: $r=0.90472$.

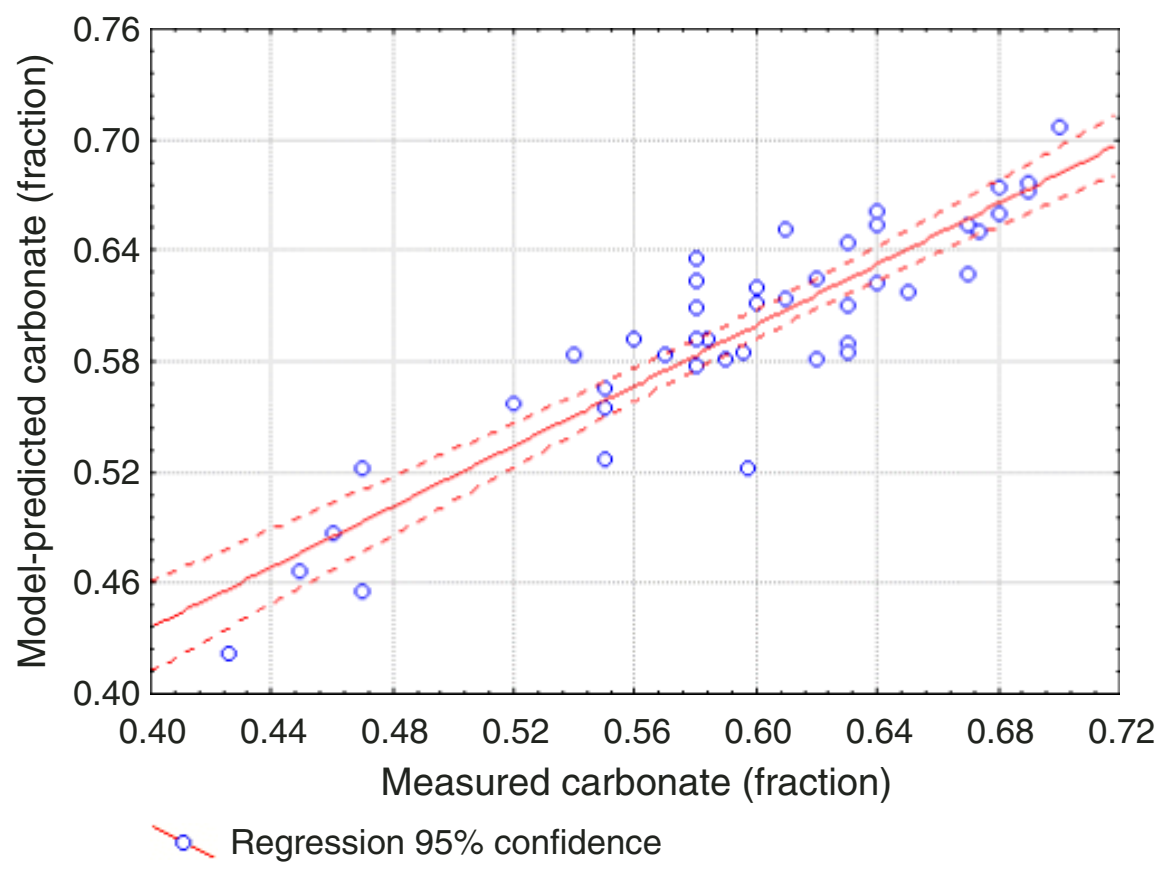


L.D. MILLWOOD ET AL.

Data Report: Color Reflectance as a Carbonate Proxy

Table ATA1. Carbonate fraction prediction model, Hole 1119C.

\begin{tabular}{|c|c|}
\hline $\begin{array}{l}\text { Carbonate fraction } \\
\text { prediction }\end{array}$ & $\begin{array}{l}\text { Depth } \\
\text { (mcd) }\end{array}$ \\
\hline 0.8893 & 0.2 \\
\hline 0.5967 & 0.3 \\
\hline 0.6104 & 0.35 \\
\hline 0.7099 & 0.4 \\
\hline 0.4192 & 0.45 \\
\hline 0.502 & 0.5 \\
\hline 0.1568 & 0.55 \\
\hline 0.1839 & 0.6 \\
\hline 0.0706 & 0.65 \\
\hline 0.0831 & 0.7 \\
\hline 0.0427 & 0.8 \\
\hline 0.0447 & 0.9 \\
\hline 0.0898 & 1 \\
\hline 0.0815 & 1.1 \\
\hline 0.0581 & 1.2 \\
\hline 0.1148 & 1.27 \\
\hline 0.0598 & 1.34 \\
\hline 0.1976 & 1.42 \\
\hline 0.2551 & 1.51 \\
\hline 0.1641 & 1.61 \\
\hline 0.143 & 1.74 \\
\hline 0.1627 & 1.84 \\
\hline 0.1123 & 1.94 \\
\hline 0.1044 & 2.03 \\
\hline 0.0496 & 2.24 \\
\hline 0.1854 & 2.34 \\
\hline 0.0854 & 2.43 \\
\hline 0.0723 & 2.54 \\
\hline 0.0796 & 2.63 \\
\hline 0.0889 & 2.72 \\
\hline 0.2784 & 2.8 \\
\hline 0.1719 & 2.9 \\
\hline 0.0884 & 3 \\
\hline 0.1009 & 3.11 \\
\hline 0.1342 & 3.2 \\
\hline 0.0909 & 3.3 \\
\hline 0.1116 & 3.4 \\
\hline 0.074 & 3.5 \\
\hline 0.0691 & 3.7 \\
\hline 0.1305 & 3.79 \\
\hline 0.4488 & 3.84 \\
\hline 0.3315 & 3.9 \\
\hline 0.1073 & 3.94 \\
\hline 0.1733 & 4 \\
\hline 0.0507 & 4.1 \\
\hline 0.1537 & 4.2 \\
\hline 0.076 & 4.3 \\
\hline 0.1725 & 4.4 \\
\hline 0.1142 & 4.5 \\
\hline 0.0541 & 4.6 \\
\hline 0.0545 & 4.7 \\
\hline 0.0275 & 4.79 \\
\hline 0.0311 & 4.89 \\
\hline 0.0294 & 4.99 \\
\hline 0.0512 & 5.09 \\
\hline 0.0347 & 5.19 \\
\hline 0.0298 & 5.24 \\
\hline 0.0303 & 5.29 \\
\hline 0.0466 & 5.34 \\
\hline 0.0982 & 5.39 \\
\hline 0.108 & 5.44 \\
\hline
\end{tabular}

Note: Only a portion of this table appears here. The complete table is available in ASCII. 
L.D. MILLWOOD ET AL.

Data Report: Color Reflectance as a Carbonate Proxy

Table ATA2. Carbonate fraction prediction model, Site 1120.

\begin{tabular}{cl}
\hline $\begin{array}{c}\text { Carbonate fraction } \\
\text { prediction }\end{array}$ & $\begin{array}{l}\text { Depth } \\
\text { (mcd) }\end{array}$ \\
\hline 0.87 & -0.22 \\
0.85 & -0.18 \\
0.83 & -0.16 \\
0.9 & -0.14 \\
0.87 & -0.12 \\
0.88 & -0.1 \\
0.86 & -0.08 \\
0.87 & -0.06 \\
0.88 & -0.04 \\
0.88 & -0.02 \\
0.86 & 0 \\
0.87 & 0.02 \\
0.88 & 0.04 \\
0.87 & 0.06 \\
0.86 & 0.08 \\
0.85 & 0.1 \\
0.86 & 0.12 \\
0.86 & 0.14 \\
0.86 & 0.16 \\
0.87 & 0.18 \\
0.88 & 0.2 \\
0.88 & 0.22 \\
0.87 & 0.24 \\
0.87 & 0.26 \\
0.87 & 0.28 \\
0.88 & 0.3 \\
0.88 & 0.32 \\
0.89 & 0.34 \\
0.89 & 0.36 \\
0.89 & 0.38 \\
0.89 & 0.4 \\
0.89 & 0.42 \\
0.86 & 0.44 \\
0.88 & 0.46 \\
0.9 & 0.48 \\
0.9 & 0.5 \\
0.9 & 0.52 \\
0.91 & 0.54 \\
0.91 & 0.56 \\
0.89 & 0.58 \\
0.88 & 0.6 \\
0.88 & 0.62 \\
0.87 & 0.64 \\
0.87 & 0.66 \\
0.87 & 0.68 \\
0.87 & 0.7 \\
0.86 & 0.72 \\
0.86 & 0.74 \\
0.86 & 0.76 \\
0.87 & 0.78 \\
0.85 & 0.8 \\
0.86 & 0.82 \\
0.86 & 0.84 \\
0.88 & 0.86 \\
0.87 & 0.88 \\
0.86 & 0.9 \\
0.86 & 0.92 \\
0.88 & 0.94 \\
0.86 & 0.96 \\
0.86 & 0.98 \\
0.87 & 1 \\
0.86 & 1.02 \\
& \\
& \\
0.96 \\
0
\end{tabular}

Note: Only a portion of this table appears here. The complete table is available in ASCII. 
L.D. MILLWOOD ET AL.

Data Report: Color Reflectance as a Carbonate Proxy

Table ATA3. Carbonate fraction prediction model, Site 1121.

\begin{tabular}{cl}
\hline $\begin{array}{c}\text { Carbonate fraction } \\
\text { prediction }\end{array}$ & $\begin{array}{l}\text { Depth } \\
\text { (mcd) }\end{array}$ \\
\hline 0.02 & 0.02 \\
0.01 & 0.04 \\
0.03 & 0.08 \\
0.15 & 0.1 \\
0.03 & 0.12 \\
0.02 & 0.14 \\
0.01 & 0.16 \\
0.01 & 0.18 \\
0.01 & 0.2 \\
0.01 & 0.22 \\
0.01 & 0.24 \\
0.01 & 0.26 \\
0.01 & 0.28 \\
0.01 & 0.3 \\
0.01 & 0.32 \\
0.01 & 0.34 \\
0.01 & 0.36 \\
0.02 & 0.38 \\
0.01 & 0.4 \\
0.01 & 0.42 \\
0.01 & 0.44 \\
0.01 & 0.46 \\
0.01 & 0.48 \\
0.01 & 0.5 \\
0.01 & 0.52 \\
0.01 & 0.54 \\
0.01 & 0.56 \\
0.01 & 0.58 \\
0.01 & 0.6 \\
0.01 & 0.62 \\
0.01 & 0.64 \\
0.01 & 0.66 \\
0.01 & 0.68 \\
0.01 & 0.7 \\
0.01 & 0.72 \\
0.01 & 0.74 \\
0.01 & 0.76 \\
0.01 & 0.78 \\
0.01 & 0.8 \\
0.01 & 0.82 \\
0.01 & 0.84 \\
0.01 & 0.86 \\
0.01 & 0.88 \\
0.01 & 0.9 \\
0.01 & 0.92 \\
0.02 & 0.94 \\
0.01 & 0.96 \\
0.01 & 0.98 \\
0.01 & 1 \\
0.01 & 1.02 \\
0.02 & 1.04 \\
0.02 & 1.06 \\
0.01 & 1.08 \\
0.01 & 1.1 \\
0.01 & 1.12 \\
0.01 & 1.14 \\
0.01 & 1.16 \\
0.01 & 1.18 \\
0.01 & 1.2 \\
0.01 & 1.22 \\
0.01 & 1.24 \\
0.01 & 1.26 \\
\hline & \\
& \\
& \\
0.01 \\
0
\end{tabular}

Note: Only a portion of this table appears here. The complete table is available in ASCII. 
L.D. MILLWOOD ET AL.

Data Report: Color Reflectance as a Carbonate Proxy

Table ATA4. Carbonate fraction prediction model, Site 1122.

\begin{tabular}{|c|c|}
\hline $\begin{array}{l}\text { Carbonate fraction } \\
\text { prediction }\end{array}$ & $\begin{array}{l}\text { Depth } \\
\text { (mcd) }\end{array}$ \\
\hline 0.18 & 0.02 \\
\hline 0.6 & 0.04 \\
\hline 0.2 & 0.06 \\
\hline 0.33 & 0.08 \\
\hline 0.18 & 0.1 \\
\hline 0.29 & 0.12 \\
\hline 0.17 & 0.14 \\
\hline 0.14 & 0.16 \\
\hline 0.17 & 0.18 \\
\hline 0.3 & 0.2 \\
\hline 0.1 & 0.22 \\
\hline 0.1 & 0.24 \\
\hline 0.17 & 0.26 \\
\hline 0.32 & 0.28 \\
\hline 0.14 & 0.3 \\
\hline 0.29 & 0.32 \\
\hline 0.33 & 0.34 \\
\hline 0.08 & 0.36 \\
\hline 0.29 & 0.38 \\
\hline 0.08 & 0.4 \\
\hline 0.05 & 0.42 \\
\hline 0.24 & 0.44 \\
\hline 0.23 & 0.46 \\
\hline 0.2 & 0.48 \\
\hline 0.1 & 0.5 \\
\hline 0.27 & 0.52 \\
\hline 0.12 & 0.54 \\
\hline 0.2 & 0.56 \\
\hline 0.09 & 0.58 \\
\hline 0.13 & 0.6 \\
\hline 0.18 & 0.62 \\
\hline 0.14 & 0.64 \\
\hline 0.15 & 0.66 \\
\hline 0.11 & 0.68 \\
\hline 0.08 & 0.7 \\
\hline 0.1 & 0.72 \\
\hline 0.51 & 0.74 \\
\hline 0.05 & 0.76 \\
\hline 0.36 & 0.78 \\
\hline 0.23 & 0.8 \\
\hline 0.11 & 0.82 \\
\hline 0.27 & 0.84 \\
\hline 0.42 & 0.86 \\
\hline 0.47 & 0.88 \\
\hline 0.35 & 0.9 \\
\hline 0.09 & 0.92 \\
\hline 0.04 & 0.94 \\
\hline 0.28 & 0.96 \\
\hline 0.21 & 0.98 \\
\hline 0.14 & 1 \\
\hline 0.08 & 1.02 \\
\hline 0.18 & 1.04 \\
\hline 0.59 & 1.06 \\
\hline 0.27 & 1.08 \\
\hline 0.13 & 1.1 \\
\hline 0.34 & 1.12 \\
\hline 0.48 & 1.14 \\
\hline 0.12 & 1.16 \\
\hline 0.26 & 1.18 \\
\hline 0.14 & 1.2 \\
\hline 0.26 & 1.22 \\
\hline 0.15 & 1.24 \\
\hline
\end{tabular}

Note: Only a portion of this table appears here. The complete table is available in ASCII. 
L.D. MILLWOOD ET AL.

Data Report: Color Reflectance as a Carbonate Proxy

Table ATA5. Carbonate fraction prediction model, Site 1123.

\begin{tabular}{|c|c|}
\hline $\begin{array}{l}\text { Carbonate fraction } \\
\text { prediction }\end{array}$ & $\begin{array}{l}\text { Depth } \\
\text { (mcd) }\end{array}$ \\
\hline 0.67 & 0.02 \\
\hline 0.63 & 0.04 \\
\hline 0.62 & 0.06 \\
\hline 0.55 & 0.08 \\
\hline 0.4 & 0.1 \\
\hline 0.49 & 0.12 \\
\hline 0.51 & 0.14 \\
\hline 0.41 & 0.16 \\
\hline 0.37 & 0.18 \\
\hline 0.43 & 0.2 \\
\hline 0.46 & 0.22 \\
\hline 0.32 & 0.24 \\
\hline 0.2 & 0.26 \\
\hline 0.24 & 0.28 \\
\hline 0.46 & 0.3 \\
\hline 0.55 & 0.32 \\
\hline 0.49 & 0.34 \\
\hline 0.57 & 0.36 \\
\hline 0.57 & 0.38 \\
\hline 0.58 & 0.4 \\
\hline 0.6 & 0.42 \\
\hline 0.56 & 0.44 \\
\hline 0.57 & 0.46 \\
\hline 0.58 & 0.48 \\
\hline 0.55 & 0.5 \\
\hline 0.52 & 0.52 \\
\hline 0.52 & 0.54 \\
\hline 0.46 & 0.56 \\
\hline 0.51 & 0.58 \\
\hline 0.5 & 0.6 \\
\hline 0.52 & 0.62 \\
\hline 0.5 & 0.64 \\
\hline 0.5 & 0.66 \\
\hline 0.51 & 0.68 \\
\hline 0.47 & 0.7 \\
\hline 0.5 & 0.72 \\
\hline 0.48 & 0.74 \\
\hline 0.49 & 0.76 \\
\hline 0.52 & 0.78 \\
\hline 0.51 & 0.8 \\
\hline 0.49 & 0.82 \\
\hline 0.51 & 0.84 \\
\hline 0.45 & 0.86 \\
\hline 0.48 & 0.88 \\
\hline 0.5 & 0.9 \\
\hline 0.48 & 0.92 \\
\hline 0.54 & 0.94 \\
\hline 0.5 & 0.96 \\
\hline 0.48 & 0.98 \\
\hline 0.48 & 1 \\
\hline 0.48 & 1.02 \\
\hline 0.5 & 1.04 \\
\hline 0.5 & 1.06 \\
\hline 0.49 & 1.08 \\
\hline 0.48 & 1.1 \\
\hline 0.46 & 1.12 \\
\hline 0.48 & 1.14 \\
\hline 0.5 & 1.16 \\
\hline 0.54 & 1.18 \\
\hline 0.49 & 1.2 \\
\hline 0.47 & 1.22 \\
\hline 0.48 & 1.24 \\
\hline
\end{tabular}

Note: Only a portion of this table appears here. The complete table is available in ASCII. 
L.D. MILLWOOD ET AL.

Data Report: Color Reflectance as a Carbonate Proxy

Table ATA6. Carbonate fraction prediction model, Hole 1123A (laboratory data).

\begin{tabular}{|c|c|c|}
\hline $\begin{array}{l}\text { Carbonate fraction } \\
\text { prediction }\end{array}$ & $\begin{array}{l}\text { Depth } \\
\text { (mcd) }\end{array}$ & $\begin{array}{l}\text { Carbonate fraction } \\
\text { measured }\end{array}$ \\
\hline 0.57 & - & 0.63 \\
\hline 0.01 & 0.03 & - \\
\hline 0.01 & 0.05 & - \\
\hline 0.03 & 0.07 & - \\
\hline 0.08 & 0.09 & - \\
\hline- & 0.1 & 0.56 \\
\hline 0.04 & 0.11 & - \\
\hline 0.02 & 0.13 & - \\
\hline 0.02 & 0.15 & - \\
\hline 0.02 & 0.17 & - \\
\hline 0.06 & 0.19 & - \\
\hline 0.06 & 0.21 & - \\
\hline 0.38 & 0.23 & - \\
\hline 0.61 & 0.25 & - \\
\hline 0.51 & 0.27 & - \\
\hline 0.54 & 0.29 & - \\
\hline 0.61 & 0.31 & - \\
\hline 0.39 & 0.33 & - \\
\hline 0.54 & 0.36 & - \\
\hline 0.49 & 0.38 & - \\
\hline 0.58 & 0.4 & - \\
\hline 0.94 & 0.42 & - \\
\hline 0.95 & 0.44 & - \\
\hline 0.91 & 0.46 & - \\
\hline 0.66 & 0.48 & - \\
\hline 0.65 & 0.5 & - \\
\hline 0.65 & 0.52 & - \\
\hline 0.67 & 0.54 & - \\
\hline 0.64 & 0.56 & - \\
\hline 0.63 & 0.58 & - \\
\hline 0.62 & 0.6 & - \\
\hline 0.53 & 0.62 & - \\
\hline 0.56 & 0.64 & - \\
\hline 0.6 & 0.66 & - \\
\hline 0.56 & 0.68 & - \\
\hline 0.62 & 0.7 & - \\
\hline 0.66 & 0.72 & - \\
\hline 0.59 & 0.74 & - \\
\hline 0.6 & 0.76 & - \\
\hline 0.58 & 0.78 & - \\
\hline 0.54 & 0.8 & - \\
\hline 0.6 & 0.82 & - \\
\hline 0.44 & 0.86 & 0.38 \\
\hline 0.57 & 0.9 & - \\
\hline 0.58 & 0.94 & - \\
\hline 0.53 & 0.96 & - \\
\hline 0.51 & 1 & - \\
\hline 0.61 & 1.04 & - \\
\hline 0.57 & 1.08 & - \\
\hline 0.56 & 1.12 & - \\
\hline 0.57 & 1.16 & - \\
\hline 0.59 & 1.2 & - \\
\hline 0.58 & 1.24 & - \\
\hline 0.56 & 1.28 & - \\
\hline 0.64 & 1.32 & - \\
\hline 0.6 & 1.36 & - \\
\hline 0.58 & 1.39 & - \\
\hline 0.32 & 1.53 & - \\
\hline 0.11 & 1.55 & - \\
\hline 0.05 & 1.57 & - \\
\hline 0.15 & 1.59 & - \\
\hline 0.61 & 1.63 & - \\
\hline 0.59 & 1.65 & - \\
\hline 0.69 & 1.68 & - \\
\hline
\end{tabular}

Note: Only a portion of this table appears here. The complete table is available in ASCII. 
L.D. MILLWOOD ET AL.

Data Report: Color Reflectance as a Carbonate Proxy

Table ATA7. Carbonate fraction prediction model, Hole 1123A (shipboard data).

\begin{tabular}{|c|c|}
\hline $\begin{array}{l}\text { Carbonate fraction } \\
\text { prediction }\end{array}$ & $\begin{array}{l}\text { Depth } \\
\text { (mcd) }\end{array}$ \\
\hline 0.69 & 0.02 \\
\hline 0.41 & 0.04 \\
\hline 0.34 & 0.06 \\
\hline 0.27 & 0.08 \\
\hline 0.44 & 0.1 \\
\hline 0.35 & 0.12 \\
\hline 0.29 & 0.14 \\
\hline 0.34 & 0.16 \\
\hline 0.32 & 0.18 \\
\hline 0.39 & 0.2 \\
\hline 0.4 & 0.22 \\
\hline 0.3 & 0.24 \\
\hline 0.27 & 0.26 \\
\hline 0.19 & 0.28 \\
\hline 0.46 & 0.3 \\
\hline 0.57 & 0.32 \\
\hline 0.44 & 0.34 \\
\hline 0.59 & 0.36 \\
\hline 0.53 & 0.38 \\
\hline 0.59 & 0.4 \\
\hline 0.62 & 0.42 \\
\hline 0.6 & 0.44 \\
\hline 0.54 & 0.46 \\
\hline 0.6 & 0.48 \\
\hline 0.62 & 0.5 \\
\hline 0.66 & 0.52 \\
\hline 0.62 & 0.54 \\
\hline 0.5 & 0.56 \\
\hline 0.61 & 0.58 \\
\hline 0.54 & 0.6 \\
\hline 0.62 & 0.62 \\
\hline 0.68 & 0.64 \\
\hline 0.54 & 0.66 \\
\hline 0.54 & 0.68 \\
\hline 0.57 & 0.7 \\
\hline 0.54 & 0.72 \\
\hline 0.53 & 0.74 \\
\hline 0.49 & 0.76 \\
\hline 0.53 & 0.78 \\
\hline 0.63 & 0.8 \\
\hline 0.54 & 0.82 \\
\hline 0.67 & 0.84 \\
\hline 0.51 & 0.86 \\
\hline 0.56 & 0.88 \\
\hline 0.63 & 0.9 \\
\hline 0.45 & 0.92 \\
\hline 0.67 & 0.94 \\
\hline 0.41 & 0.96 \\
\hline 0.52 & 0.98 \\
\hline 0.54 & 1 \\
\hline 0.57 & 1.02 \\
\hline 0.62 & 1.04 \\
\hline 0.52 & 1.06 \\
\hline 0.59 & 1.08 \\
\hline 0.63 & 1.1 \\
\hline 0.48 & 1.12 \\
\hline 0.65 & 1.14 \\
\hline 0.56 & 1.16 \\
\hline 0.52 & 1.18 \\
\hline 0.68 & 1.2 \\
\hline 0.59 & 1.22 \\
\hline 0.57 & 1.24 \\
\hline
\end{tabular}

Note: Only a portion of this table appears here. The complete table is available in ASCII. 
L.D. MILLWOOD ET AL.

Data Report: Color Reflectance as a Carbonate Proxy

Table ATA8. Carbonate fraction prediction model, Site 1124.

\begin{tabular}{cl}
\hline $\begin{array}{c}\text { Carbonate fraction } \\
\text { prediction }\end{array}$ & $\begin{array}{l}\text { Depth } \\
\text { (mcd) }\end{array}$ \\
\hline 0.63 & 0.02 \\
0.85 & 0.04 \\
0.62 & 0.06 \\
0.51 & 0.08 \\
0.22 & 0.1 \\
0.39 & 0.12 \\
0.37 & 0.14 \\
0.54 & 0.16 \\
0.76 & 0.18 \\
0.47 & 0.2 \\
0.67 & 0.22 \\
0.73 & 0.24 \\
0.67 & 0.26 \\
0.75 & 0.28 \\
0.76 & 0.3 \\
0.88 & 0.32 \\
0.76 & 0.34 \\
0.61 & 0.36 \\
0.35 & 0.38 \\
0.29 & 0.4 \\
0.51 & 0.42 \\
0.41 & 0.44 \\
0.49 & 0.46 \\
0.11 & 0.48 \\
0.08 & 0.5 \\
0.11 & 0.52 \\
0.25 & 0.54 \\
0.31 & 0.56 \\
0.22 & 0.58 \\
0.25 & 0.6 \\
0.41 & 0.62 \\
0.22 & 0.64 \\
0.29 & 0.66 \\
0.14 & 0.68 \\
0.42 & 0.7 \\
0.37 & 0.72 \\
0.19 & 0.74 \\
0.23 & 0.76 \\
0.18 & 0.78 \\
0.38 & 0.8 \\
0.47 & 0.82 \\
0.19 & 0.84 \\
0.27 & 0.86 \\
0.28 & 0.88 \\
0.16 & 0.9 \\
0.31 & 0.92 \\
0.15 & 0.94 \\
0.29 & 0.96 \\
0.21 & 0.98 \\
0.13 & 1 \\
0.27 & 1.02 \\
0.21 & 1.04 \\
0.23 & 1.06 \\
0.14 & 1.08 \\
0.14 & 1.1 \\
0.21 & 1.12 \\
0.11 & 1.14 \\
0.28 & 1.16 \\
0.24 & 1.18 \\
0.15 & 1.2 \\
0.19 & 1.22 \\
0.26 & 1.24 \\
\hline & \\
\hline & \\
& \\
& \\
0.25 \\
0
\end{tabular}

Note: Only a portion of this table appears here. The complete table is available in ASCII. 
L.D. MILLWOOD ET AL.

Data Report: Color Reflectance as a Carbonate Proxy

Table ATA9. Carbonate fraction prediction model, Site 1125.

\begin{tabular}{|c|c|}
\hline $\begin{array}{l}\text { Carbonate fraction } \\
\text { prediction }\end{array}$ & $\begin{array}{l}\text { Depth } \\
\text { (mcd) }\end{array}$ \\
\hline 0.48 & 0.02 \\
\hline 0.52 & 0.04 \\
\hline 0.49 & 0.06 \\
\hline 0.43 & 0.08 \\
\hline 0.46 & 0.1 \\
\hline 0.51 & 0.12 \\
\hline 0.49 & 0.14 \\
\hline 0.47 & 0.16 \\
\hline 0.41 & 0.18 \\
\hline 0.53 & 0.2 \\
\hline 0.44 & 0.22 \\
\hline 0.46 & 0.24 \\
\hline 0.44 & 0.26 \\
\hline 0.41 & 0.28 \\
\hline 0.51 & 0.3 \\
\hline 0.47 & 0.32 \\
\hline 0.53 & 0.34 \\
\hline 0.47 & 0.36 \\
\hline 0.41 & 0.38 \\
\hline 0.43 & 0.4 \\
\hline 0.45 & 0.42 \\
\hline 0.38 & 0.44 \\
\hline 0.4 & 0.46 \\
\hline 0.48 & 0.48 \\
\hline 0.52 & 0.5 \\
\hline 0.42 & 0.52 \\
\hline 0.41 & 0.54 \\
\hline 0.45 & 0.56 \\
\hline 0.45 & 0.58 \\
\hline 0.34 & 0.6 \\
\hline 0.41 & 0.62 \\
\hline 0.33 & 0.64 \\
\hline 0.3 & 0.66 \\
\hline 0.25 & 0.68 \\
\hline 0.41 & 0.7 \\
\hline 0.35 & 0.72 \\
\hline 0.22 & 0.74 \\
\hline 0.3 & 0.76 \\
\hline 0.22 & 0.78 \\
\hline 0.37 & 0.8 \\
\hline 0.44 & 0.82 \\
\hline 0.46 & 0.84 \\
\hline 0.49 & 0.86 \\
\hline 0.38 & 0.88 \\
\hline 0.34 & 0.9 \\
\hline 0.53 & 0.92 \\
\hline 0.39 & 0.94 \\
\hline 0.53 & 0.96 \\
\hline 0.49 & 0.98 \\
\hline 0.53 & 1 \\
\hline 0.38 & 1.02 \\
\hline 0.48 & 1.04 \\
\hline 0.47 & 1.06 \\
\hline 0.48 & 1.08 \\
\hline 0.5 & 1.1 \\
\hline 0.46 & 1.12 \\
\hline 0.47 & 1.14 \\
\hline 0.48 & 1.16 \\
\hline 0.47 & 1.18 \\
\hline 0.48 & 1.2 \\
\hline 0.46 & 1.22 \\
\hline 0.49 & 1.24 \\
\hline
\end{tabular}

Note: Only a portion of this table appears here. The complete table is available in ASCII. 
L.D. MILLWOOD ET AL.

Data Report: Color Reflectance as a Carbonate Proxy

Table ATD1. Carbonate model parameter estimates, Site 1119C (laboratory reflectance data).

\begin{tabular}{|c|c|c|c|c|c|}
\hline Variable & DF & $\begin{array}{l}\text { Parameter } \\
\text { estimate }\end{array}$ & $\begin{array}{l}\text { Standard } \\
\text { error }\end{array}$ & $t$ value & $\begin{array}{c}\text { Probability } \\
>|t|\end{array}$ \\
\hline Intercept & 1 & -2.99843 & 0.94981 & -3.16 & 0.0055 \\
\hline FD1 & 1 & -0.98097 & 1.56765 & -0.63 & 0.5393 \\
\hline FD2 & 1 & 2.72693 & 3.78007 & 0.72 & 0.4799 \\
\hline FD3 & 1 & 3.23423 & 4.25149 & 0.76 & 0.4567 \\
\hline FD4 & 1 & -5.3057 & 5.092 & -1.04 & 0.3112 \\
\hline FD5 & 1 & 5.55867 & 5.35941 & 1.04 & 0.3134 \\
\hline FD6 & 1 & -9.14216 & 4.74102 & -1.93 & 0.0697 \\
\hline FD7 & 1 & 4.52201 & 7.66529 & 0.59 & 0.5626 \\
\hline FD8 & 1 & -9.91156 & 10.99747 & -0.9 & 0.3794 \\
\hline FD9 & 1 & 9.92222 & 8.07165 & 1.23 & 0.2348 \\
\hline FD10 & 1 & -6.57747 & 6.63406 & -0.99 & 0.3346 \\
\hline FD11 & 1 & 13.7145 & 7.52135 & 1.82 & 0.0849 \\
\hline FD12 & 1 & -11.73328 & 11.11737 & -1.06 & 0.3052 \\
\hline FD13 & 1 & -8.6031 & 11.90562 & -0.72 & 0.4792 \\
\hline FD14 & 1 & 34.98892 & 16.2556 & 2.15 & 0.0452 \\
\hline FD15 & 1 & -43.54069 & 14.98702 & -2.91 & 0.0094 \\
\hline FD16 & 1 & 34.10985 & 15.85578 & 2.15 & 0.0453 \\
\hline FD17 & 1 & -29.81858 & 14.65664 & -2.03 & 0.0569 \\
\hline FD18 & 1 & 19.69968 & 12.98729 & 1.52 & 0.1467 \\
\hline FD19 & 1 & -3.4008 & 12.46584 & -0.27 & 0.7881 \\
\hline FD20 & 1 & 3.99621 & 10.82892 & 0.37 & 0.7164 \\
\hline FD21 & 1 & -12.93237 & 7.88485 & -1.64 & 0.1183 \\
\hline FD22 & 1 & 13.22727 & 8.89713 & 1.49 & 0.1544 \\
\hline FD23 & 1 & -3.6155 & 7.53423 & -0.48 & 0.6371 \\
\hline FD24 & 1 & 3.03142 & 9.13306 & 0.33 & 0.7438 \\
\hline FD25 & 1 & 2.5092 & 8.87236 & 0.28 & 0.7805 \\
\hline FD26 & 1 & -6.16231 & 7.48504 & -0.82 & 0.4211 \\
\hline FD27 & 1 & 5.69712 & 6.54848 & 0.87 & 0.3958 \\
\hline FD28 & 1 & -9.50305 & 5.5104 & -1.72 & 0.1017 \\
\hline FD29 & 1 & 8.45407 & 7.82123 & 1.08 & 0.294 \\
\hline FD30 & 1 & -9.08062 & 7.32659 & -1.24 & 0.2311 \\
\hline FD31 & 1 & 4.86389 & 4.46034 & 1.09 & 0.2899 \\
\hline
\end{tabular}

Notes: $F D=$ first derivative of the spectral value, $D F=$ degrees of freedom. This table is also available in ASCII. 
L.D. MILLWOOD ET AL.

Data Report: Color Reflectance as a Carbonate Proxy

Table ATD2. Carbonate model parameter estimates, Site 1120.

\begin{tabular}{lcrcrr}
\hline Variable & DF & \multicolumn{1}{c}{$\begin{array}{c}\text { Parameter } \\
\text { estimate }\end{array}$} & $\begin{array}{c}\text { Standard } \\
\text { error }\end{array}$ & $t$ value & $\begin{array}{c}\text { Probability } \\
>|t|\end{array}$ \\
\hline Intercept & 1 & -3.070736 & 0.36716485 & -8.363 & 0.0001 \\
R1 & 1 & 0.009393 & 0.00257489 & 3.648 & 0.0008 \\
FD2 & 1 & 0.167758 & 0.18876931 & 0.889 & 0.3795 \\
FD7 & 1 & -0.251446 & 0.11494365 & -2.188 & 0.0346 \\
FD21 & 1 & 0.962151 & 0.44639092 & 2.155 & 0.0372 \\
FD27 & 1 & 0.612194 & 0.32778476 & 1.868 & 0.0691 \\
\hline
\end{tabular}

Notes: $F D=$ first derivative of the spectral value, $D F=$ degrees of freedom. This table is also available in ASCII. 
L.D. MILLWOOD ET AL.

Data Report: Color Reflectance as a Carbonate Proxy

Table ATD3. Carbonate model parameter estimates, Site 1121.

\begin{tabular}{lccccc}
\hline Variable & DF & $\begin{array}{c}\text { Parameter } \\
\text { estimate }\end{array}$ & $\begin{array}{c}\text { Standard } \\
\text { error }\end{array}$ & $t$ value & $\begin{array}{c}\text { Probability } \\
>|t|\end{array}$ \\
\hline Intercept & 1 & -5.620012 & 0.42901373 & -13.1 & 0.0001 \\
R1 & 1 & 0.16317 & 0.03494793 & 4.669 & 0.0003 \\
FD4 & 1 & 1.135263 & 1.75661072 & 0.646 & 0.5279 \\
FD7 & 1 & -0.763586 & 1.41667175 & -0.539 & 0.5978 \\
FD21 & 1 & -1.828062 & 1.15902038 & -1.577 & 0.1356 \\
FD30 & 1 & 0.220452 & 1.61975973 & 0.136 & 0.8936 \\
\hline
\end{tabular}

Notes: $F D=$ first derivative of the spectral value, $D F=$ degrees of freedom. This table is also available in ASCII. 
L.D. MILLWOOD ET AL.

Data Report: Color Reflectance as a Carbonate Proxy

Table ATD4. Carbonate model parameter estimates, Site 1122.

\begin{tabular}{|c|c|c|c|c|c|}
\hline Variable & DF & $\begin{array}{c}\text { Parameter } \\
\text { estimate }\end{array}$ & $\begin{array}{l}\text { Standard } \\
\text { error }\end{array}$ & $t$ value & $\begin{array}{c}\text { Probability } \\
>|t|\end{array}$ \\
\hline Intercept & 1 & -3.468443 & 0.34917308 & -9.933 & 0.0001 \\
\hline $\mathrm{R} 1$ & 1 & 0.083495 & 0.03199619 & 2.61 & 0.0102 \\
\hline FD1 & 1 & -0.135564 & 1.09738822 & -0.124 & 0.9019 \\
\hline FD2 & 1 & 0.385547 & 1.25895955 & 0.306 & 0.7599 \\
\hline FD3 & 1 & 2.843244 & 1.67442428 & 1.698 & 0.092 \\
\hline FD4 & 1 & -6.050862 & 1.93205619 & -3.132 & 0.0022 \\
\hline FD5 & 1 & 3.270204 & 2.08845871 & 1.566 & 0.1199 \\
\hline FD6 & 1 & 1.546564 & 2.39147613 & 0.647 & 0.519 \\
\hline FD7 & 1 & -1.827609 & 2.65128781 & -0.689 & 0.4919 \\
\hline FD8 & 1 & 6.306459 & 2.91249788 & 2.165 & 0.0323 \\
\hline FD9 & 1 & -0.130816 & 3.62342379 & -0.036 & 0.9713 \\
\hline FD10 & 1 & -1.997526 & 3.67184398 & -0.544 & 0.5874 \\
\hline FD11 & 1 & -4.331576 & 3.61102073 & -1.2 & 0.2326 \\
\hline FD12 & 1 & -0.657204 & 2.31582148 & -0.284 & 0.777 \\
\hline FD13 & 1 & 3.693833 & 2.74086755 & 1.348 & 0.1802 \\
\hline FD14 & 1 & 3.766126 & 2.99810836 & 1.256 & 0.2114 \\
\hline FD15 & 1 & 0.598826 & 2.80640415 & 0.213 & 0.8314 \\
\hline FD16 & 1 & -2.192557 & 2.76860924 & -0.792 & 0.4299 \\
\hline FD17 & 1 & -4.819435 & 3.06717936 & -1.571 & 0.1186 \\
\hline FD18 & 1 & -1.86988 & 3.03179697 & -0.617 & 0.5385 \\
\hline FD19 & 1 & -2.719279 & 3.32958889 & -0.817 & 0.4157 \\
\hline FD20 & 1 & 4.32702 & 3.12336881 & 1.385 & 0.1684 \\
\hline FD21 & 1 & 4.353374 & 3.04718516 & 1.429 & 0.1556 \\
\hline FD22 & 1 & 5.845123 & 3.09968423 & 1.886 & 0.0617 \\
\hline FD23 & 1 & 3.662064 & 3.29173707 & 1.113 & 0.2681 \\
\hline FD24 & 1 & 3.939838 & 2.92208429 & 1.348 & 0.18 \\
\hline FD25 & 1 & 2.067159 & 2.45690793 & 0.841 & 0.4017 \\
\hline FD26 & 1 & -5.19903 & 2.19128415 & -2.373 & 0.0192 \\
\hline FD27 & 1 & -3.364824 & 1.87781259 & -1.792 & 0.0756 \\
\hline FD28 & 1 & -5.120462 & 2.73008164 & -1.876 & 0.063 \\
\hline FD29 & 1 & -0.528119 & 1.9072034 & -0.277 & 0.7823 \\
\hline FD30 & 1 & 0.016276 & 1.66908074 & 0.01 & 0.9922 \\
\hline
\end{tabular}

Notes: $F D=$ first derivative of the spectral value, $D F=$ degrees of freedom. This table is also available in ASCII. 
L.D. MILLWOOD ET AL.

Data Report: Color Reflectance as a Carbonate Proxy

Table ATD5. Carbonate model parameter estimates, Site 1123.

\begin{tabular}{|c|c|c|c|c|c|}
\hline Variable & DF & $\begin{array}{l}\text { Parameter } \\
\text { estimate }\end{array}$ & $\begin{array}{l}\text { Standard } \\
\text { error }\end{array}$ & $t$ value & $\begin{array}{c}\text { Probability } \\
\quad>|t|\end{array}$ \\
\hline Intercept & 1 & -0.270424 & 0.179995 & -1.502 & 0.1338 \\
\hline $\mathrm{R} 1$ & 1 & 0.034378 & 0.00715145 & 4.807 & 0.0001 \\
\hline FD1 & 1 & 0.147009 & 0.31077197 & 0.473 & 0.6364 \\
\hline FD2 & 1 & 0.504987 & 0.38425196 & 1.314 & 0.1895 \\
\hline FD3 & 1 & 0.42146 & 0.58730695 & 0.718 & 0.4734 \\
\hline FD4 & 1 & -0.868137 & 0.69533258 & -1.249 & 0.2126 \\
\hline FD5 & 1 & 0.851732 & 0.69010051 & 1.234 & 0.2179 \\
\hline FD6 & 1 & -0.398603 & 0.72015026 & -0.553 & 0.5802 \\
\hline FD7 & 1 & -0.497294 & 0.94275553 & -0.527 & 0.5982 \\
\hline FD8 & 1 & 2.001106 & 0.88258588 & 2.267 & 0.0239 \\
\hline FD9 & 1 & -1.517632 & 1.01126583 & -1.501 & 0.1342 \\
\hline FD10 & 1 & 0.898916 & 1.20886784 & 0.744 & 0.4576 \\
\hline FD11 & 1 & 0.757887 & 1.08772666 & 0.697 & 0.4864 \\
\hline FD12 & 1 & -0.319059 & 0.76216745 & -0.419 & 0.6757 \\
\hline FD13 & 1 & 0.040624 & 0.98258152 & 0.041 & 0.967 \\
\hline FD14 & 1 & -0.687926 & 1.02189399 & -0.673 & 0.5012 \\
\hline FD15 & 1 & -2.819029 & 0.88760059 & -3.176 & 0.0016 \\
\hline FD16 & 1 & -0.526675 & 0.74935174 & -0.703 & 0.4826 \\
\hline FD17 & 1 & -0.932751 & 0.96872366 & -0.963 & 0.3362 \\
\hline FD18 & 1 & 0.93326 & 1.1162914 & 0.836 & 0.4036 \\
\hline FD19 & 1 & 1.501798 & 0.95615302 & 1.571 & 0.1171 \\
\hline FD20 & 1 & 1.753132 & 1.01384471 & 1.729 & 0.0846 \\
\hline FD21 & 1 & 1.400305 & 0.94039255 & 1.489 & 0.1373 \\
\hline FD22 & 1 & 1.237253 & 0.94922837 & 1.303 & 0.1932 \\
\hline FD23 & 1 & 2.186203 & 0.96462098 & 2.266 & 0.024 \\
\hline FD24 & 1 & 0.59675 & 0.80103779 & 0.745 & 0.4567 \\
\hline FD25 & 1 & -1.255316 & 0.70015999 & -1.793 & 0.0738 \\
\hline FD26 & 1 & -1.305811 & 0.6826952 & -1.913 & 0.0565 \\
\hline FD27 & 1 & -1.425399 & 0.63202426 & -2.255 & 0.0247 \\
\hline FD28 & 1 & -1.321559 & 0.64191962 & -2.059 & 0.0402 \\
\hline FD29 & 1 & -0.992825 & 0.5537088 & -1.793 & 0.0737 \\
\hline FD30 & 1 & 0.422484 & 0.5292249 & 0.798 & 0.4252 \\
\hline
\end{tabular}

Notes: $F D=$ first derivative of the spectral value, $D F=$ degrees of freedom. This table is also available in ASCII. 
L.D. MILLWOOD ET AL.

Data Report: Color Reflectance as a Carbonate Proxy

Table ATD6. Carbonate model parameter estimates, Hole 1123A (laboratory reflectance data).

\begin{tabular}{lcclcc}
\hline & & Parameter & Standard & Probability \\
Variable & DF & estimate & error & $t$ value & $>|t|$ \\
\hline Intercept & 1 & 0.397657 & 0.84062297 & 0.473 & 0.6388 \\
FD1 & 1 & -1.221498 & 1.17201929 & -1.042 & 0.3037 \\
FD2 & 1 & 0.99481 & 1.39209148 & 0.715 & 0.4791 \\
FD3 & 1 & 0.15499 & 2.30908722 & 0.067 & 0.9468 \\
FD4 & 1 & -1.444551 & 2.49973134 & -0.578 & 0.5667 \\
FD5 & 1 & 0.743232 & 3.16172009 & 0.235 & 0.8154 \\
FD6 & 1 & -1.539407 & 3.0980059 & -0.497 & 0.622 \\
FD7 & 1 & -2.610672 & 2.79101746 & -0.935 & 0.3553 \\
FD8 & 1 & -0.004683 & 3.07132697 & -0.002 & 0.9988 \\
FD9 & 1 & 2.880878 & 3.11523161 & 0.925 & 0.3608 \\
FD10 & 1 & 1.141798 & 3.26149783 & 0.35 & 0.7282 \\
FD11 & 1 & 1.090231 & 4.0960116 & 0.266 & 0.7915 \\
FD12 & 1 & 2.68583 & 4.73281187 & 0.567 & 0.5736 \\
FD13 & 1 & -0.367905 & 5.89596151 & -0.062 & 0.9506 \\
FD14 & 1 & 3.946258 & 5.6831952 & 0.694 & 0.4916 \\
FD15 & 1 & -5.103774 & 4.76727138 & -1.071 & 0.2909 \\
FD16 & 1 & -0.810301 & 5.59102084 & -0.145 & 0.8855 \\
FD17 & 1 & -4.802781 & 4.07568571 & -1.178 & 0.2458 \\
FD18 & 1 & -0.531941 & 4.08093122 & -0.13 & 0.897 \\
FD19 & 1 & -0.249661 & 4.04838269 & -0.062 & 0.9511 \\
FD20 & 1 & -3.919904 & 3.85363489 & -1.017 & 0.3153 \\
FD21 & 1 & 0.750353 & 4.07868506 & 0.184 & 0.855 \\
FD22 & 1 & -3.457597 & 3.31511884 & -1.043 & 0.3034 \\
FD23 & 1 & -0.655458 & 3.58226358 & -0.183 & 0.8558 \\
FD24 & 1 & -2.817448 & 3.11082491 & -0.906 & 0.3707 \\
FD25 & 1 & 3.314245 & 2.83920737 & 1.167 & 0.2502 \\
FD26 & 1 & 0.482871 & 3.03967398 & 0.159 & 0.8746 \\
FD27 & 1 & 4.516534 & 2.95377925 & 1.529 & 0.1343 \\
FD28 & 1 & 0.362925 & 3.01158018 & 0.121 & 0.9047 \\
FD29 & 1 & 3.031825 & 2.49775621 & 1.214 & 0.2321 \\
FD30 & 1 & -1.283941 & 2.27758193 & -0.564 & 0.5762 \\
\hline & & & & & \\
\hline
\end{tabular}

Notes: $F D=$ first derivative of the spectral value, $D F=$ degrees of freedom. This table is also available in ASCII. 
L.D. MILLWOOD ET AL.

Data Report: Color Reflectance as a Carbonate Proxy

Table ATD7. Carbonate model parameter estimates, Hole 1123A (shipboard reflectance data).

\begin{tabular}{|c|c|c|c|c|c|}
\hline Variable & $\mathrm{DF}$ & $\begin{array}{l}\text { Parameter } \\
\text { estimate }\end{array}$ & $\begin{array}{l}\text { Standard } \\
\text { error }\end{array}$ & $t$ value & $\begin{array}{c}\text { Probability } \\
\qquad|t|\end{array}$ \\
\hline Intercept & 1 & 0.096174 & 0.29577128 & 0.325 & 0.7456 \\
\hline FD1 & 1 & 0.120709 & 0.43741023 & 0.276 & 0.783 \\
\hline FD2 & 1 & 0.300916 & 0.63450718 & 0.474 & 0.6361 \\
\hline FD3 & 1 & 0.649694 & 0.88053544 & 0.738 & 0.4619 \\
\hline FD4 & 1 & -1.922469 & 1.14770208 & -1.675 & 0.0962 \\
\hline FD5 & 1 & 1.180058 & 1.08041375 & 1.092 & 0.2766 \\
\hline FD6 & 1 & 0.781749 & 1.21724002 & 0.642 & 0.5218 \\
\hline FD7 & 1 & -1.504981 & 1.4632074 & -1.029 & 0.3055 \\
\hline FD8 & 1 & 2.831768 & 1.66048252 & 1.705 & 0.0904 \\
\hline FD9 & 1 & -1.429901 & 1.71100763 & -0.836 & 0.4048 \\
\hline FD10 & 1 & -0.432801 & 2.05420943 & -0.211 & 0.8334 \\
\hline FD11 & 1 & 0.925894 & 1.54548818 & 0.599 & 0.5501 \\
\hline FD12 & 1 & -0.493676 & 1.08875475 & -0.453 & 0.651 \\
\hline FD13 & 1 & 1.328391 & 1.87057004 & 0.71 & 0.4788 \\
\hline FD14 & 1 & -3.709336 & 1.71597415 & -2.162 & 0.0324 \\
\hline FD15 & 1 & 0.015332 & 1.37631024 & 0.011 & 0.9911 \\
\hline FD16 & 1 & 0.349213 & 1.40752424 & 0.248 & 0.8044 \\
\hline FD17 & 1 & -1.836055 & 1.77608393 & -1.034 & 0.3031 \\
\hline FD18 & 1 & 0.245988 & 1.81486539 & 0.136 & 0.8924 \\
\hline FD19 & 1 & -0.162188 & 1.66083628 & -0.098 & 0.9223 \\
\hline FD20 & 1 & 1.63303 & 1.74323059 & 0.937 & 0.3505 \\
\hline FD21 & 1 & 2.033068 & 1.70997161 & 1.189 & 0.2365 \\
\hline FD22 & 1 & 0.866931 & 1.53790022 & 0.564 & 0.5739 \\
\hline FD23 & 1 & 1.475374 & 1.496568 & 0.986 & 0.326 \\
\hline FD24 & 1 & -2.048453 & 1.23542738 & -1.658 & 0.0996 \\
\hline FD25 & 1 & 0.466575 & 1.10738094 & 0.421 & 0.6742 \\
\hline FD26 & 1 & -0.812477 & 1.04076368 & -0.781 & 0.4364 \\
\hline FD27 & 1 & -0.658182 & 0.93611871 & -0.703 & 0.4832 \\
\hline FD28 & 1 & -0.315403 & 0.95027493 & -0.332 & 0.7405 \\
\hline FD29 & 1 & -0.369535 & 0.70991543 & -0.521 & 0.6035 \\
\hline FD30 & 1 & 0.119638 & 0.80239094 & 0.149 & 0.8817 \\
\hline
\end{tabular}

Notes: $F D=$ first derivative of the spectral value, $D F=$ degrees of freedom. This table is also available in ASCII. 
L.D. MILLWOOD ET AL.

Data Report: Color Reflectance as a Carbonate Proxy

Table ATD8. Carbonate model parameter estimates, Site 1124.

\begin{tabular}{lccccc}
\hline & & Parameter & Standard & & Probability \\
Variable & DF & Estimate & Error & $t$ value & $>|t|$ \\
\hline Intercept & 1 & -3.070736 & 0.36716485 & -8.363 & 0.0001 \\
FD1 & 1 & -0.778838 & 0.74158287 & -1.05 & 0.2952 \\
FD2 & 1 & 0.351576 & 0.91793439 & 0.383 & 0.7022 \\
FD3 & 1 & 1.359917 & 1.35766851 & 1.002 & 0.3181 \\
FD4 & 1 & -0.964937 & 1.78744175 & -0.54 & 0.5901 \\
FD5 & 1 & 4.362448 & 1.79543135 & 2.43 & 0.0163 \\
FD6 & 1 & 3.264014 & 1.81658741 & 1.797 & 0.0743 \\
FD7 & 1 & 3.189519 & 2.14711187 & 1.485 & 0.1394 \\
FD8 & 1 & -2.017352 & 2.26028484 & -0.893 & 0.3735 \\
FD9 & 1 & -2.107639 & 2.53158599 & -0.833 & 0.4064 \\
FD10 & 1 & -8.041198 & 2.60156451 & -3.091 & 0.0024 \\
FD11 & 1 & -0.052052 & 2.38323222 & -0.022 & 0.9826 \\
FD12 & 1 & 1.536598 & 1.64428331 & 0.935 & 0.3515 \\
FD13 & 1 & 3.534009 & 2.23453252 & 1.582 & 0.1158 \\
FD14 & 1 & -0.74144 & 1.98946557 & -0.373 & 0.7099 \\
FD15 & 1 & 4.030173 & 2.58674006 & 1.558 & 0.1213 \\
FD16 & 1 & -4.36457 & 1.99025894 & -2.193 & 0.0298 \\
FD17 & 1 & -2.977724 & 1.81793244 & -1.638 & 0.1035 \\
FD18 & 1 & 0.829916 & 2.26907548 & 0.366 & 0.715 \\
FD19 & 1 & 0.984388 & 2.31003007 & 0.426 & 0.6706 \\
FD20 & 1 & 5.654193 & 2.64363589 & 2.139 & 0.034 \\
FD21 & 1 & 5.377573 & 2.3121821 & 2.326 & 0.0213 \\
FD22 & 1 & 3.341636 & 2.04371563 & 1.635 & 0.1041 \\
FD23 & 1 & 0.209657 & 2.12629549 & 0.099 & 0.9216 \\
FD24 & 1 & -0.471768 & 1.84290502 & -0.256 & 0.7983 \\
FD25 & 1 & -0.365236 & 1.94388952 & -0.188 & 0.8512 \\
FD26 & 1 & -2.700741 & 1.59603491 & -1.692 & 0.0926 \\
FD27 & 1 & -2.812718 & 1.6927878 & -1.662 & 0.0986 \\
FD28 & 1 & -2.59178 & 1.4100281 & -1.838 & 0.068 \\
FD29 & 1 & -4.158899 & 1.31412081 & -3.165 & 0.0019 \\
FD30 & 1 & -1.470326 & 1.2570415 & -1.17 & 0.2439 \\
\hline & & & & & \\
\hline
\end{tabular}

Notes: $F D=$ first derivative of the spectral value, $D F=$ degrees of freedom. This table is also available in ASCII. 
L.D. MILLWOOD ET AL.

Data Report: Color Reflectance as a Carbonate Proxy

Table ATD9. Carbonate model parameter estimates, Site 1125.

\begin{tabular}{|c|c|c|c|c|c|}
\hline Variable & DF & $\begin{array}{l}\text { Parameter } \\
\text { estimate }\end{array}$ & $\begin{array}{l}\text { Standard } \\
\text { error }\end{array}$ & $t$ value & $\begin{array}{c}\text { Probability } \\
\quad>|t|\end{array}$ \\
\hline Intercept & 1 & -0.533222 & 0.34716475 & -1.536 & 0.1454 \\
\hline FD1 & 1 & -0.008915 & 0.53909035 & -0.017 & 0.987 \\
\hline FD2 & 1 & -0.95887 & 0.68953505 & -1.391 & 0.1846 \\
\hline FD3 & 1 & -0.497242 & 0.87731722 & -0.567 & 0.5792 \\
\hline FD4 & 1 & -0.02663 & 1.14091026 & -0.023 & 0.9817 \\
\hline FD5 & 1 & 0.970385 & 1.04939154 & 0.925 & 0.3698 \\
\hline FD6 & 1 & -0.138252 & 1.05208208 & -0.131 & 0.8972 \\
\hline FD7 & 1 & 2.96633 & 1.39258388 & 2.13 & 0.0501 \\
\hline FD8 & 1 & -3.003108 & 1.63565197 & -1.836 & 0.0863 \\
\hline FD9 & 1 & 1.138776 & 1.57997197 & 0.721 & 0.4821 \\
\hline FD10 & 1 & -1.068847 & 1.72886105 & -0.618 & 0.5457 \\
\hline FD11 & 1 & 1.179204 & 1.78867083 & 0.659 & 0.5197 \\
\hline FD12 & 1 & -0.667715 & 1.58137318 & -0.422 & 0.6788 \\
\hline FD13 & 1 & -0.437562 & 1.18243757 & -0.37 & 0.7165 \\
\hline FD14 & 1 & 0.472936 & 1.78633436 & 0.265 & 0.7948 \\
\hline FD15 & 1 & -0.976412 & 1.78999581 & -0.545 & 0.5934 \\
\hline FD16 & 1 & -0.225967 & 2.09762238 & -0.108 & 0.9156 \\
\hline FD17 & 1 & -1.148753 & 1.65150713 & -0.696 & 0.4973 \\
\hline FD18 & 1 & -2.481787 & 1.51318137 & -1.64 & 0.1218 \\
\hline FD19 & 1 & 0.355226 & 1.88324854 & 0.189 & 0.8529 \\
\hline FD20 & 1 & 0.004327 & 2.17949542 & 0.002 & 0.9984 \\
\hline FD21 & 1 & 0.542901 & 2.02117108 & 0.269 & 0.7919 \\
\hline FD22 & 1 & -0.564499 & 1.4515264 & -0.389 & 0.7028 \\
\hline FD23 & 1 & -2.320998 & 1.23461516 & -1.88 & 0.0797 \\
\hline FD24 & 1 & -0.747813 & 1.08050416 & -0.692 & 0.4995 \\
\hline FD25 & 1 & 1.300399 & 0.84504229 & 1.539 & 0.1447 \\
\hline FD26 & 1 & 0.065563 & 0.88835153 & 0.074 & 0.9421 \\
\hline FD27 & 1 & 1.176387 & 1.10359386 & 1.066 & 0.3033 \\
\hline FD28 & 1 & 0.508425 & 0.95661447 & 0.531 & 0.6029 \\
\hline FD29 & 1 & 0.236891 & 0.97661569 & 0.243 & 0.8116 \\
\hline FD30 & 1 & 1.165451 & 1.21492436 & 0.959 & 0.3526 \\
\hline
\end{tabular}

Notes: $F D=$ first derivative of the spectral value, $D F=$ degrees of freedom. This table is also available in ASCII. 